\title{
TU/e EnNHONEN

\section{Modeling rarefied gas-solid surface interactions for Couette flow with different wall temperatures using an unsupervised machine learning technique}

\section{Citation for published version (APA):}

Mohammad Nejad, S., lype, E., Nedea, S., Frijns, A., \& Smeulders, D. (2021). Modeling rarefied gas-solid surface interactions for Couette flow with different wall temperatures using an unsupervised machine learning technique. Physical Review E, 104(1-2), [015309]. https://doi.org/10.1103/PhysRevE.104.015309

DOI:

10.1103/PhysRevE.104.015309

Document status and date:

Published: 21/07/2021

\section{Document Version:}

Publisher's PDF, also known as Version of Record (includes final page, issue and volume numbers)

\section{Please check the document version of this publication:}

- A submitted manuscript is the version of the article upon submission and before peer-review. There can be important differences between the submitted version and the official published version of record. People interested in the research are advised to contact the author for the final version of the publication, or visit the DOI to the publisher's website.

- The final author version and the galley proof are versions of the publication after peer review.

- The final published version features the final layout of the paper including the volume, issue and page numbers.

Link to publication

\footnotetext{
General rights

- You may freely distribute the URL identifying the publication in the public portal. follow below link for the End User Agreement:

www.tue.nl/taverne

\section{Take down policy}

If you believe that this document breaches copyright please contact us at:

openaccess@tue.nl

providing details and we will investigate your claim.
}

Copyright and moral rights for the publications made accessible in the public portal are retained by the authors and/or other copyright owners and it is a condition of accessing publications that users recognise and abide by the legal requirements associated with these rights.

- Users may download and print one copy of any publication from the public portal for the purpose of private study or research.

- You may not further distribute the material or use it for any profit-making activity or commercial gain

If the publication is distributed under the terms of Article 25fa of the Dutch Copyright Act, indicated by the "Taverne" license above, please 


\title{
Modeling rarefied gas-solid surface interactions for Couette flow with different wall temperatures using an unsupervised machine learning technique
}

\author{
Shahin Mohammad Nejad $\odot,{ }^{1}$ Eldhose Iype $\odot,{ }^{2}$ Silvia Nedea, ${ }^{1}$ Arjan Frijns $\odot,{ }^{1, *}$ and David Smeulders ${ }^{1}$ \\ ${ }^{1}$ Department of Mechanical Engineering, Eindhoven University of Technology, P.O. Box 513, 5600 MB Eindhoven, The Netherlands \\ ${ }^{2}$ Department of Chemical Engineering, BITS Pilani, Dubai Campus, International Academic City, Dubai, UAE
}

(Received 11 March 2021; accepted 17 June 2021; published 21 July 2021)

\begin{abstract}
In rarefied gas flows, discontinuity phenomena such as velocity slip and temperature jump commonly appear in the gas layer adjacent to a solid boundary. Due to the physical complexity of the interactions at the gas-solid interface, particularly in the case of systems with local nonequilibrium state, boundary models with limited number of parameters cannot completely describe the reflection of gas molecules at the boundary. In this work, the Gaussian mixture (GM) model, which is an unsupervised machine learning technique, is employed to construct a statistical gas-solid surface scattering model based on the collisional data obtained from molecular dynamics (MD) simulations. The GM model is applied to study Couette flow for different inert gases (Ar and $\mathrm{He}$ ) confined between two parallel infinite gold walls at different temperatures. A direct comparison between the results obtained from the GM model and the Cercignani-Lampis-Lord (CLL) scattering kernel against the MD collisional data in terms of the distribution of the predicted postcollisional velocities, and accommodation coefficients has shown that the results from the GM model are an excellent match with the MD results outperforming the CLL scattering kernel. As an example, for He gas, while the predicted energy accommodation coefficient by the CLL model is more than two times higher than the MD predictions, the value computed by the GM model is in excellent agreement with the MD results. This superior performance of the GM model confirms its high potential to derive a generalized boundary condition in systems encountered with highly nonequilibrium and complex gas flow conditions.
\end{abstract}

DOI: 10.1103/PhysRevE.104.015309

\section{INTRODUCTION}

Heat and momentum transfer of fluid flows in contact with surfaces in noncontinuum or rarefied conditions has been an active research topic in numerous modern engineering applications such as vacuum technology, micro or nanoelectromechanical systems (M/NEMS), astronautics, and particle sizing techniques used in the aerosol industry [1-4]. In these systems jump conditions for temperature and velocity, which manifest themselves mainly at the gas-solid interfaces, are of crucial importance in computing the drag force and heat transfer experienced at the solid surfaces. To simulate the fluid flow in such systems under the moderately rarefied condition, that means a Knudsen number $(\mathrm{Kn})$ less than 0.1, the Navier Stokes (NS) equations may be used but slip boundary conditions should be considered. However, for a higher degree of rarefaction $(\mathrm{Kn}>0.1)$ the NS equations break down and must be substituted by more sophisticated equations such as Boltzmann equations. Computational-particle-based simulation techniques such as direct simulation Monte Carlo (DSMC) [5], lattice Boltzmann method (LBM) [6], and method of moments (MoM) [7] are commonly employed to find approximate solutions to the different forms of the Boltzmann equation. Nevertheless, in all these simulation techniques, rigorous prescription of boundary conditions at

\footnotetext{
*a.j.h.frijns@tue.nl
}

solid surfaces is the crucial parameter to guarantee the reliability of the simulation results. Although the gas-surface interaction (GSI) has been long studied [8-10], and a wide range of numerical models have been developed to describe it, a generalized formalism applicable in varied applications is still missing. The most well-known available GSI models are phenomenological models expressed by scattering kernels, which are probability density functions (PDF) correlating the velocity distributions of the gas molecules before and after colliding with the solid surface.

The Maxwell model [8] is the oldest empirical GSI model. Maxwell postulated that a fraction of the incident gas molecules was reflected in a diffuse manner, while the remaining part underwent a specular reflection. Despite its simplicity and acceptable accuracy, the Maxwell model was incapable of reproducing the lobular patterns observed in the molecular beam experiment [11]. Later on, to fulfill this shortcoming, Cercignani and Lampis [9] developed a more elaborate scattering kernel, which was extended further by Lord [10], and showed better performance compared to the Maxwell model [12].The Maxwell and Cercignani-Lampis-Lord (CLL) models are controlled by one or several constant parameters known as accommodation coefficients (ACs), which quantify the energy and momentum exchange at the gas-solid interface and must be known a priori. In fact, evaluating ACs is the major bottleneck in the performance of the aforementioned empirical scattering models. This is due to the superposition of many factors affecting GSI at the microscopic level, which causes a notable discrepancy among the reported values for 
ACs in experimental and numerical studies [13-16]. Several investigations [12,17-19] assessed the performance of the existing empirical GSI models. Considering hard assumptions like, for instance, the usage of constant ACs for all impinging gas molecules, assuming fixed functional relationships between the momentum and energy ACs, as well as neglecting the interplay between different components of gas molecule velocities, these models do not fully capture the physics behind the gas-wall scattering process. Moreover, these models are not capable of capturing the flow properties in a highly nonequilibrium state, frequently present in M/NEMS. This involves, for instance, impinging or reflecting molecular gas fluxes for complex flow conditions and for complex interfacial structures and conditions $[17,20]$. To construct more reliable scattering kernels some researchers extended the classical GSI models. For instance, Struchtrup [21] incorporated a velocity dependent AC with classical Maxwell scattering kernel. To describe gas scattering adjacent to an anisotropic surface, Dadzie and Meolans [22], as well as To et al. [23] proposed the usage of different ACs in each spatial direction. However, these boundary models, due to higher number of tunable parameters, are very complex to implement, and therefore have not been employed much in practice. To study Couette flow using DSMC simulations Yamamoto et al. [24], adapted the Maxwell model by using different directional ACs to generate each velocity component. Nevertheless, it was shown that the velocity correlations obtained from the Yamamoto model can still be very different from the explicit wall simulations [25].

A promising approach to achieve a detailed understanding about GSI at the atomistic level is molecular dynamics (MD) simulations, which has been successfully interplayed with DSMC to study rarefied gas flow [26-28]. However, it is well known that MD simulations are computationally expensive. A new class of scattering kernels, known as nonparametric scattering kernels, were proposed in the literature [29-31]. The main idea behind developing such models is to overcome some intrinsic limits of the existing empirical GSI models, which are mainly caused by employing a limited number of fitting parameters (i.e., accommodation coefficients). Generally speaking, in these models collision data obtained by MD simulations are directly used to construct the scattering kernel and no additional parameters such as ACs are needed to be computed in between. Assessment of the postcollisional velocities predicted by nonparametric GSI models against the results obtained from the classical parametric GSI models, MD simulations results, as well as experimental studies confirm the superior performance of the nonparametric GSI models.

Recently a new type of GSI model using the Gaussian mixture (GM) model, which is a robust unsupervised machine learning technique, was proposed by Liao et al. [32]. In the aforementioned study, for training the GM model the collisional data obtained from the molecular beam MD setup were utilized, in which only gas-wall interactions were taken into account, and gas-gas interactions were ignored. The main advantage of using the GM model over the classical scattering kernels is that since it does not require the use of any ACs as input parameters, it maintains all the important physics included in the collisional data. Investigating both smooth and

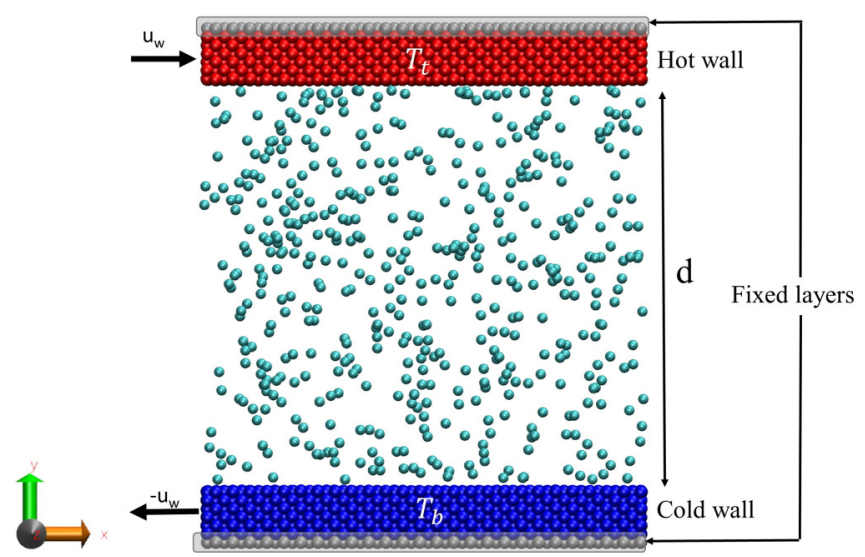

FIG. 1. Schematic representation of the system under consideration for the MD simulations; $d$ : distance between the two walls; $T_{b}$ : Temperature of the bottom wall; $T_{t}$ : Temperature of the top wall; $u_{w}$ : Imposed velocity on the walls.

rough atomic surfaces, they reported a good performance of the GM model in predicting physical and statistical properties of the gas-wall interfaces. However, it was shown that in many microgas flow systems the adsorption of gas molecules on solid surfaces impacts the gas scattering from the surfaces [33]. In the presence of the gas adsorbed layer, both the gas-gas and gas-wall interactions are not negligible. To our knowledge, no GM-based model exists that includes gas flows, internal gas-gas interactions, and highly nonequilibrium effects.

The main aim of the present paper is to investigate the capability of the GM model in the study of the rarefied gas-solid surface interactions in the case of systems encountered with highly nonequilibrium and flow conditions. Herein, considering that both gas-gas and gas-wall interactions can influence the gas flow solution in the vicinity of a solid wall, a nanochannel system consisting of two infinite parallel plates with $\mathrm{Ar}$ and $\mathrm{He}$ gases confined between them is chosen as a case study. A Couette flow was used to study the influence of the wall velocity on the residence time of the gas molecules in the interface region and the molecular scattering effects. Two types of systems, with walls at the same temperature and with walls at different temperatures, are studied using MD simulations. In each case, the performance of the GM model is evaluated in comparison with the CLL model, as well as the original MD-obtained collision data. The evaluation is carried out in terms of correlations between gas molecules's velocities before and after colliding with the surface, the PDF of the postcollisional gas velocities, and predicted ACs.

\section{METHODOLOGIES}

\section{A. MD simulation}

The three-dimensional schematic diagram of the MD simulation setup is shown in Fig. 1. The system model consists of two parallel walls including of $8750 \mathrm{Au}$ atoms each with either $800 \mathrm{Ar}$ or $400 \mathrm{He}$ atoms confined between them. In each wall $\mathrm{Au}$ atoms are arranged in a FCC lattice structure with a cross section area of $10 \mathrm{~nm}$ by $10 \mathrm{~nm}$. The normal distance 
TABLE I. Lennard-Jones potential parameters.

\begin{tabular}{lcl}
\hline \hline Atom pair & $\epsilon(\mathrm{meV})$ & $\sigma(\AA)$ \\
\hline $\mathrm{Ar}-\mathrm{Au}$ & 11.36 & 3.819 \\
$\mathrm{He}-\mathrm{Au}$ & 0.787 & 4.342 \\
$\mathrm{Ar}-\mathrm{Ar}$ & 12.2 & 3.35 \\
$\mathrm{He}-\mathrm{He}$ & 0.94 & 2.64 \\
\hline \hline
\end{tabular}

between the walls $d$ is fixed at $12 \mathrm{~nm}$ for Ar and $102 \mathrm{~nm}$ for He gas. The number of gas atoms confined between the walls and the distance between the walls were chosen in a way that the Knudsen number remains in the early transition regime $(0.1<K n<1)$, and the pressure in the gas domain does not overpass the critical pressure $\left(P_{c r}\right)$ value in the system (for Ar: $P_{c r}=4.89 \mathrm{MPa}$, and for $\mathrm{He}: P_{c r}=0.23 \mathrm{MPa}$ [34]). Therefore, for the Ar-Au system the Knudsen number and the reduced density $(\eta)$ are $K n=0.2$ and $\eta=0.02$, respectively. In addition, for the $\mathrm{He}-\mathrm{Au}$ system $K n=0.71$ and $\eta=0.00045$, respectively. A Berendsen thermostat with a damping constant of $100 \mathrm{fs}$ is employed to maintain walls temperature at the desired values. Regarding the gas atoms, initially their temperature is set at the $300 \mathrm{~K}$. Afterwards, gas atoms are in a microcanonical ensemble (NVE) and their temperature can only evolve through collisions with other atoms in the simulation box.

In this system, periodic boundary conditions are applied in the directions parallel to the wall $(x, z)$ such that only a section of an infinite wall has to be modeled. The interaction between $\mathrm{Au}$ atoms are modeled by the embedded atom model (EAM) potential [35]. The gas-gas and gas-solid interactions are modeled by the Lennard-Jones (LJ) potential and the potential parameters are listed in Table I. It is noteworthy to mention that in the atomistic study of the GSI, the pair potential parameters employed to describe the interaction strength at the gas-solid interface are of prominent importance [36,37]. It has been shown that the interaction potentials based on mixing rules cannot accurately describe GSI, and the quantum calculations based on pair potentials are the most reliable ones to study the GSI at the molecular scale $[36,38,39]$. Therefore, in this work the gas-wall interaction potential parameters are calculated based on existing quantum $a b$ initio computations [40]. To model the Couette flow condition, walls have been moving with the relative velocity difference of $2 u_{w}$ (see Fig. 1). The speed ratio $S_{w}$ is defined as the ratio between the wall velocity $u_{w}$ and the most probable speed of the gas at the temperature $T_{b}: S_{w}=\frac{u_{w}}{\sqrt{2 k_{B} T_{b} / m_{g}}}$, where $k_{B}$ is the Boltzmann constant, $T_{b}$ is the temperature of the bottom wall, and $m_{g}$ is the mass of the gas atom.

In all the simulations, after performing the initial surface energy minimization, the MD setup has been equilibrated at the desired temperature for $3 \mathrm{~ns}$ using the time step of $\Delta t=1 \mathrm{fs}$ and $0.5 \mathrm{fs}$ for Ar-Au and He-Au systems, respectively. Once the system is fully equilibrated the production run is started. To guarantee reliable statistics it is proceeded for the next $25 \mathrm{~ns}$ and $100 \mathrm{~ns}$ for $\mathrm{Ar}-\mathrm{Au}$ and $\mathrm{He}-\mathrm{Au}$ systems, respectively. More details regarding the gathering collisional data based on the discussed MD simulation setup can be found in our previous work [36]. All the MD simulations were performed using the LAMMPS [41] molecular dynamics package.

\section{B. CLL scattering kernel}

When considering the flow of a dilute gas adjacent to a solid surface, prescribing the proper boundary condition is the key factor for obtaining a reliable solution of the Boltzmann equation. In other words, it is of crucial importance to know how gas molecules impinging with certain velocity with a surface will be reflected from it. In kinetic theory analysis, the gas-surface interaction models are employed as boundary conditions for the Boltzmann equation. The GSI model, also called the scattering kernel, is defined by the conditional probability density function $f\left(\boldsymbol{v} \mid \boldsymbol{v}^{\prime}\right)$, which represents the probability density that an impinging gas molecule with velocity $\boldsymbol{v}^{\prime}$ is rebounced with velocity $\boldsymbol{v}$ from the surface. The CLL model is the most accurate phenomenological gassurface collision model because of its relatively well-defined mathematical framework leading to the satisfactory prediction of the scattering process. In the CLL model the gas molecule velocity components in the tangential $\left(v_{t}\right)$ and normal $\left(v_{n}\right)$ directions are assumed to be independent from each other, and they can be determined through Eqs. (1) and (2), respectively, as follows:

$$
\begin{gathered}
f^{\mathrm{CLL}}\left(v_{t} \mid v_{t}^{\prime}\right)=\frac{1}{\sqrt{\pi \alpha_{t}\left(2-\alpha_{t}\right)}} \exp -\frac{\left[v_{t}-\left(1-\alpha_{t}\right) v_{t}^{\prime}\right]^{2}}{\alpha_{t}\left(2-\alpha_{t}\right)}, \\
f^{\mathrm{CLL}}\left(v_{n} \mid v_{n}^{\prime}\right)=\frac{2 v_{n}}{\alpha_{N E}} \exp \left[-\frac{v_{n}^{2}+\left(1-\alpha_{N E}\right) v_{n}^{\prime 2}}{\alpha_{N E}}\right] I_{0} \\
\times\left[\frac{2\left(\sqrt{1-\alpha_{N E}}\right) v_{n} v_{n}^{\prime}}{\alpha_{N E}}\right],
\end{gathered}
$$

where $\alpha_{t}$ and $\alpha_{N E}$ are the accommodation coefficients corresponding to the tangential momentum and normal kinetic energy, respectively. $I_{0}$ denotes the modified Bessel function of the first order and zeroth order. Here the velocities are normalized by $\sqrt{2 R T_{w}}$, where $R$ is the specific gas constant $\left(\frac{k_{B}}{m_{g}}\right)$ and $T_{w}$ is the wall temperature. In this model, a fixed empirical correlation is imposed between $\alpha_{t}$ and the tangential energy accommodation coefficient $\alpha_{\mathrm{TE}}$, that is, $\alpha_{\mathrm{TE}}=\alpha_{t}(2-$ $\alpha_{t}$ ). To generate new velocities after collision according to the CLL scattering kernel the algorithm provided in the paper by Peddakotla et al. [42] is employed, in which including a separate $\mathrm{AC}$ for each spatial direction assists the comparison with MD simulation results.

Accommodation coefficients used normally to characterize the energy and momentum exchange in the gas-solid interface are the main input parameters required in the CLL model. In this work, to compute ACs the method proposed by Spijker et al. [25], which also allows evaluating ACs for an isothermal system, is employed. In this approach, the slope of the best least-squares linear fit of the MD obtained collisional data is used for computing various ACs

$$
\alpha_{q}=1-\frac{\sum_{i}\left(Q_{I}^{i}-\left\langle Q_{I}\right\rangle\right)\left(Q_{R}^{i}-\left\langle Q_{R}\right\rangle\right)}{\sum_{i}\left(Q_{I}^{i}-\left\langle Q_{I}\right\rangle\right)^{2}},
$$

where subscript $q$ can refer to various gas molecule kinematic properties such as its momentum or kinetic energy in 
a certain direction. $Q_{I}^{i}$ and $Q_{R}^{i}$ represent the precollisional and postcollisional value of the same property for the gas particle, respectively. The notation $\langle$.$\rangle indicates the ensemble average$ of the molecular property $Q$.

\section{Gaussian mixture model}

The Gaussian mixture model is an unsupervised learning method, which found its applications in speech recognition [43], image, and pattern recognition [44,45] among many other areas. Generally, the GM model is categorized as a parametric model, but due to the presence of the numerous fitting parameters in the model it retains the flexibility of a nonparametric model. Therefore, it can be considered as a promising tool to model high-dimensional data space. The idea of representing data as a collection of $D$-dimensional Gaussians ( $D$ being the number of features) is desirable in its application to predict scattering velocity distributions, which are also mostly Gaussians. A GM model of the $N_{G}$ component Gaussians with mean vector $\vec{\mu}_{i}$ and covariance matrix $\Sigma_{i}$ is given by Eq. (4)

$$
p(\vec{x})=\sum_{i=1}^{N_{G}} w_{i} g\left(\vec{x} \mid \vec{\mu}_{i}, \Sigma_{i}\right)
$$

where

$$
\begin{aligned}
g\left(\vec{x} \mid \vec{\mu}_{i}, \Sigma_{i}\right)= & \frac{1}{(2 \pi)^{D / 2}\left|\Sigma_{i}\right|^{1 / 2}} \exp \\
& \times\left[-\frac{1}{2}\left(\vec{x}-\vec{\mu}_{i}\right)^{\prime} \Sigma_{i}^{-1}\left(\vec{x}-\vec{\mu}_{i}\right)\right] .
\end{aligned}
$$

The parameters $\left(\left\{w_{i}, \vec{\mu}_{i}, \Sigma_{i}\right\} \forall i\right.$ in $\left.\left\{1 \cdots N_{G}\right\}\right)$ in the model are optimized using the expectation-maximization (EM) algorithm [46]. All calculations in this work used the GM model package available in SCIKIT-LEARN [47] with all default settings except for the number of Gaussians $\left(N_{G}\right)$.
In fact, in the case of training a GM model $N_{G}$ is the only parameter, which needs to be assigned by the user. It plays the role of a fitting parameter in the model and it has a direct impact on the performance of the model. Therefore, to prevent the occurrence of overfitting or underfitting in the model defining an optimal value of $N_{G}$ is crucial. A detailed discussion on the effect of $N_{G}$ is provided in Sec. III A. Here the entire collisional data (i.e., pre and postcollision velocities) obtained from MD simulations are considered as the training data for the GM model. This means the interplay between different velocity components is already included in the model. After training the model, using the optimum model parameters $\left(w_{i}, \vec{\mu}_{i}, \Sigma_{i}\right)$, the conditional form of the scattering kernel required by coarse grained simulation techniques can be obtained as a mixture of Gaussian functions.

\section{RESULTS AND DISCUSSION}

\section{A. Different approaches for feeding the training data into Gaussian mixture model}

Considering the collision data obtained from MD simulations, the tangential velocity components $\left(v_{x}^{\prime}, v_{z}^{\prime}, v_{x}, v_{z}\right)$ follow Gaussian distributions. However, the normal velocity components $\left(v_{y}^{\prime}, v_{y}\right)$ follow a Rayleigh distribution. In Ref. [32] the authors suggested a preprocessing scheme (see Scheme-I in Fig. 2). They mainly employed such a scheme to transfer the normal velocity components from Rayleigh distribution to a Gaussian distribution to obtain the same distribution as the tangential velocity components. This leads to a consistency in the overall distribution (Gaussian) with the basis sets (also Gaussian) in the GM model. For this purpose, initially for each velocity pair $\left(\boldsymbol{v}^{\prime}, \boldsymbol{v}\right)$ an implicit pair $\left(-\boldsymbol{v}^{\prime}, \boldsymbol{- v}\right)$ was added to the data set. Afterwards, implementing the transfer function proposed in Ref. [32] and given in

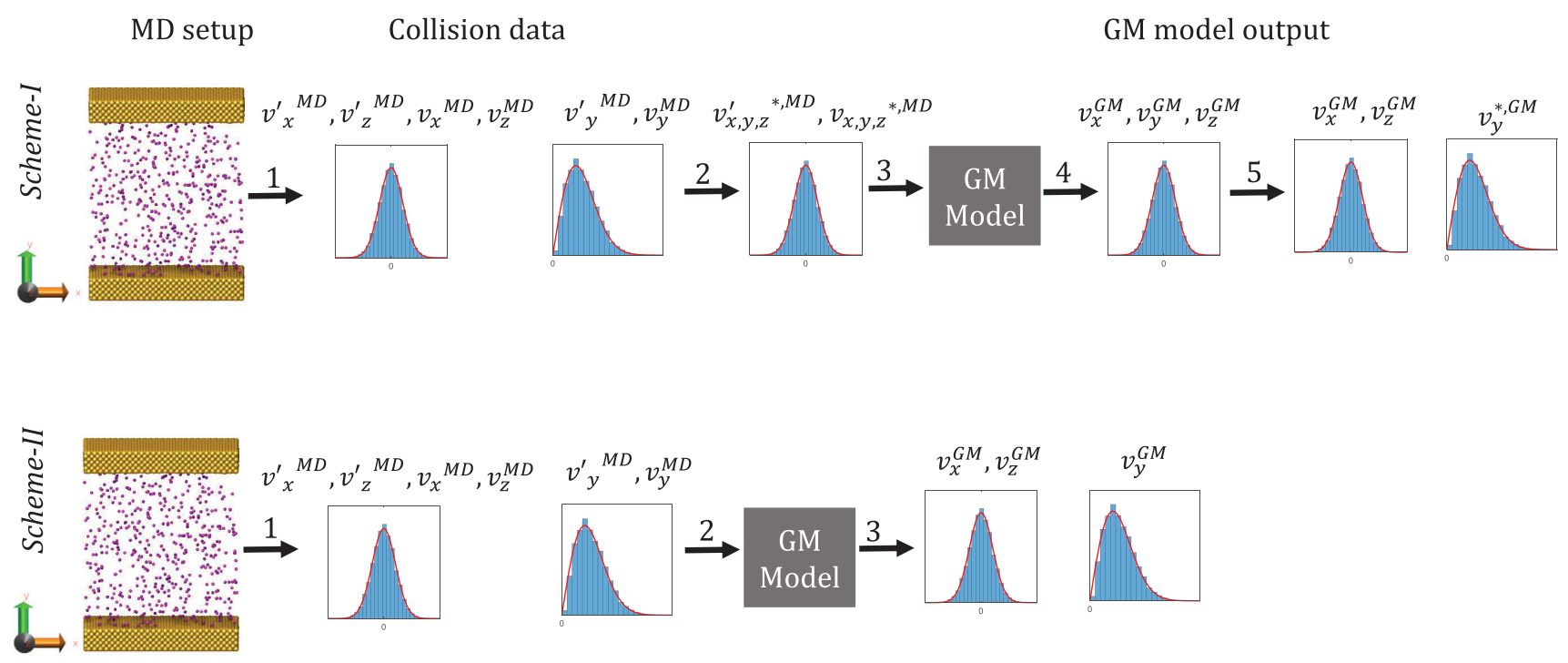

FIG. 2. Different schemes used to feed the collision data obtained by MD simulations into the GM model; $v_{x}^{\prime \mathrm{MD}}, v_{y}^{\mathrm{MD}}, v_{z}^{\mathrm{MD}}, v_{x}^{\mathrm{MD}}, v_{y}^{\mathrm{MD}}$, and $v_{z}^{\mathrm{MD}}$ are velocity components obtained from MD simulation, respectively; $v_{x, y, z}^{*, \mathrm{MD}}$ and $v_{x, y, z}^{*, \mathrm{MD}}$ are transformed incoming and outgoing velocity vectors obtained from MD simulations; $v_{x}^{\mathrm{GM}}, v_{y}^{\mathrm{GM}}$, and $v_{z}^{\mathrm{GM}}$ are velocity components predicted by GM model; $v_{y}^{*, \mathrm{GM}}$ is the normal velocity component predicted by the GM model and mapped back into the Rayleigh distribution. 

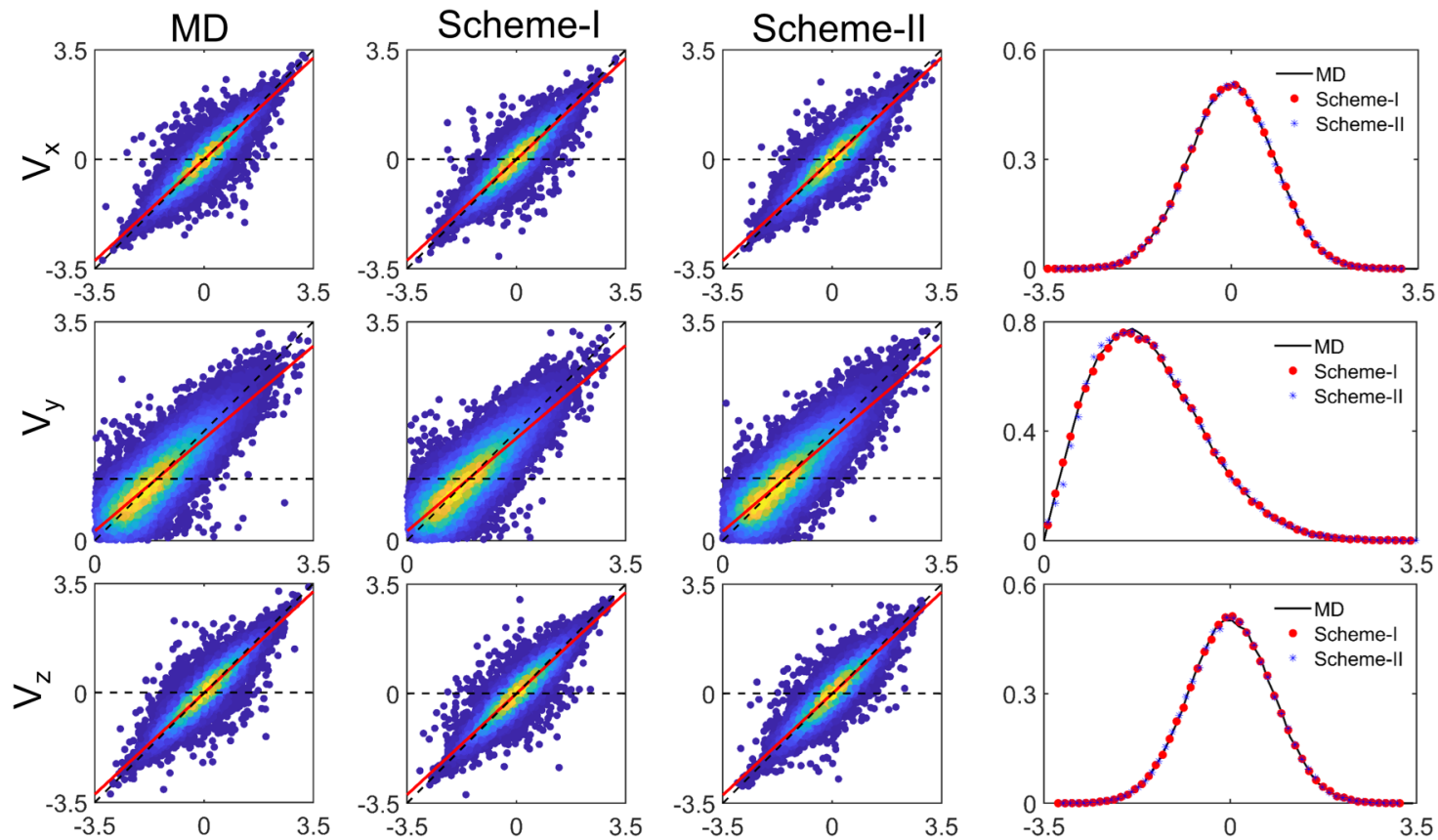

FIG. 3. Velocity correlations of impinging ( $x$ axis) and reflected ( $y$ axis) velocity components both in $\mathrm{nm} / \mathrm{ps}$ for He-Au system. The dashed horizontal and diagonal lines indicate fully diffusive and specular conditions, respectively. Red lines indicate the least-square linear fit of the data. In the last column the corresponding probability density function for reflecting particles are shown. Scheme-I uses preprocessed MD results. Scheme-II uses MD results without any preprocessing.

Eq. (5) (step 2 in Scheme-I) results in the Gaussian distributions for the normal velocity components $\left(v_{y}^{\prime *, \mathrm{MD}}, v_{y}^{*, \mathrm{MD}}\right)$

$$
T^{-1}(\zeta)=\sqrt{-2 \beta \ln \left[\frac{1}{2}-\frac{1}{2} \operatorname{erf}\left(\frac{\zeta}{\sqrt{2 \beta}}\right)\right]}
$$

$$
T(\zeta)=\sqrt{2 \beta} \operatorname{erf}^{-1}\left[1-2 \exp \left(-\frac{\zeta^{2}}{2 \beta}\right)\right], \quad \beta=\frac{k_{B} T_{g}}{m_{g}}
$$

where $T_{g}$ is the gas temperature and can be computed from the average gas kinetic energy.
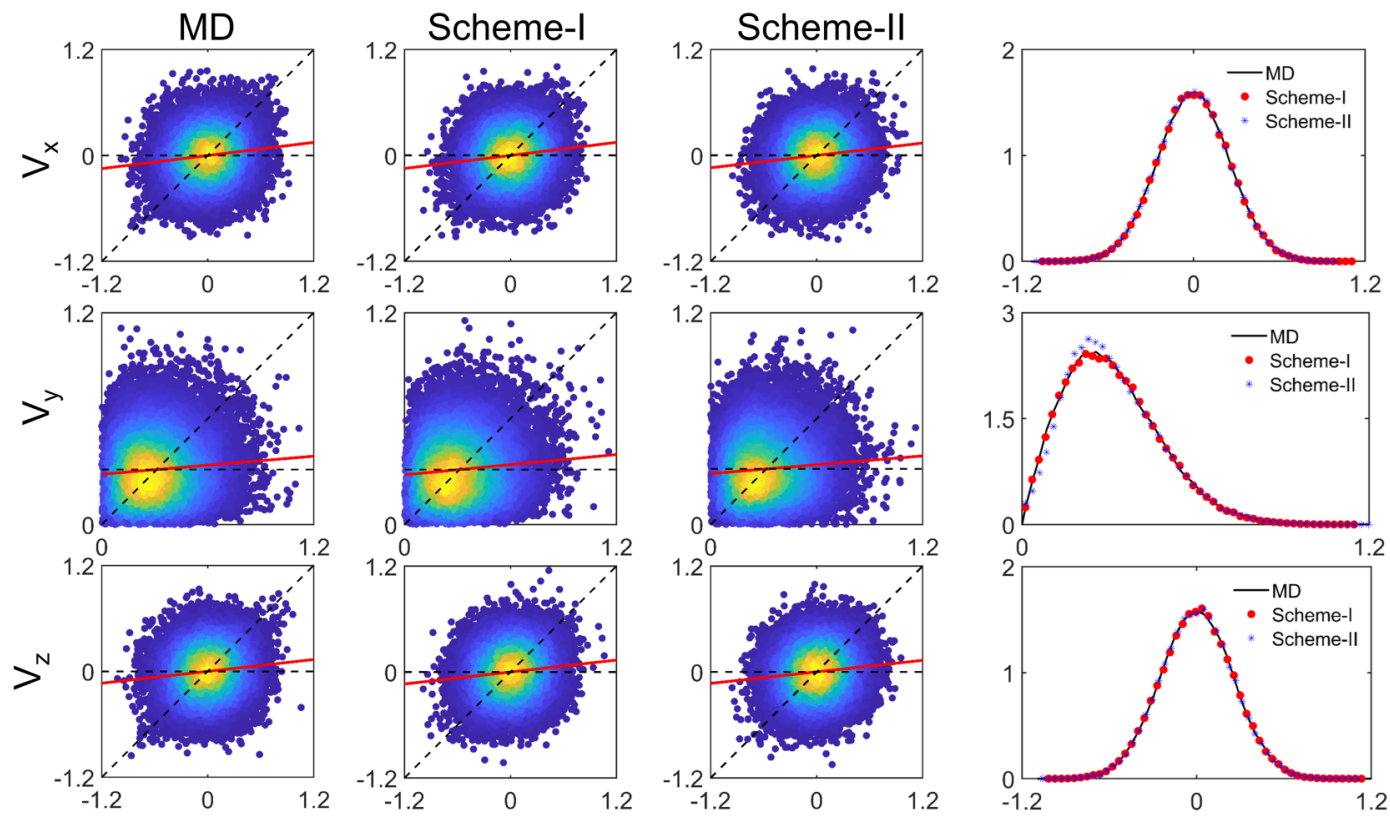

FIG. 4. Velocity correlations of impinging ( $x$ axis) and reflected ( $y$ axis) velocity components both in $\mathrm{nm} / \mathrm{ps}$ for Ar-Au system. The dashed horizontal and diagonal lines indicate fully diffusive and specular conditions, respectively. Red lines indicate the least-square linear fit of the data. In the last column the corresponding probability density function for reflecting particles has been shown. Scheme-I uses preprocessed MD results. Scheme-II uses MD results without any preprocessing. 


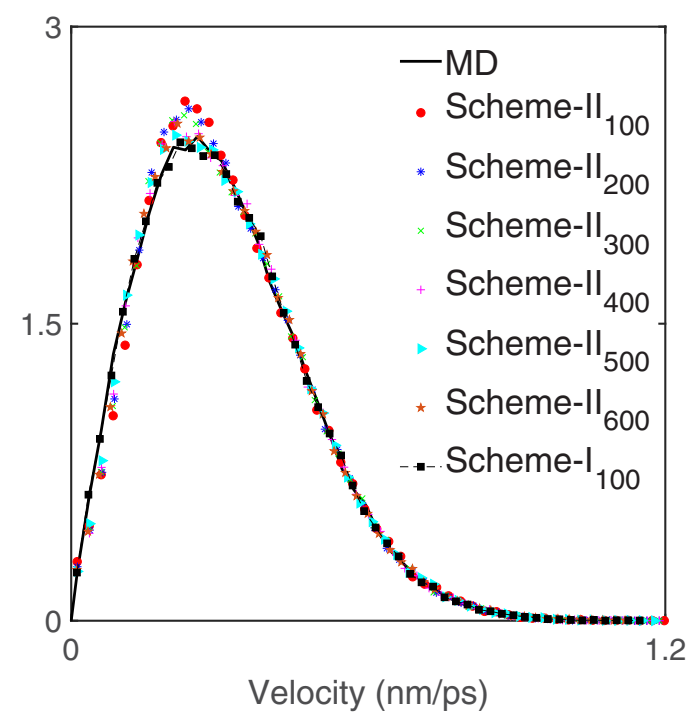

FIG. 5. Impact of the number of Gaussian functions $\left(N_{G}\right)$ in the predicted velocity distribution in the normal direction by GM model in the case of Ar-Au system. Scheme- $I_{100}$ uses preprocessed MD results based on $N_{G}=100$. Scheme- $I I_{100 \leqslant N_{G} \leqslant 600}$ uses MD results without any preprocessing.

In Ref. [32], it was mentioned that applying the introduced preprocessing step on the input data leads to a better performance of the GM model. However, it was not clarified which were the key factors considered to play a role in analyzing the GM model performance. Having a purpose slightly different than the one in Ref. [32], that is, in applying the GM model to study the correlation between impinging and outgoing velocity components in each direction rather than for overall kinetic energy, it was important to analyze the impact of applying the transfer function in different steps on the molecular scattering results for different directions. Therefore, to investigate the impact of the proposed scheme on the performance of the GM model in our system, two different approaches for implementing the collision data obtained by MD simulations were employed. In the first case (Scheme-I), preconditioning was carried out before logging the collision data into the GM model. In the second case (Scheme-II), as it is depicted in Fig. 2, MD simulations's collisional data were directly fed into the GM model without using any preprocessing. It is note-
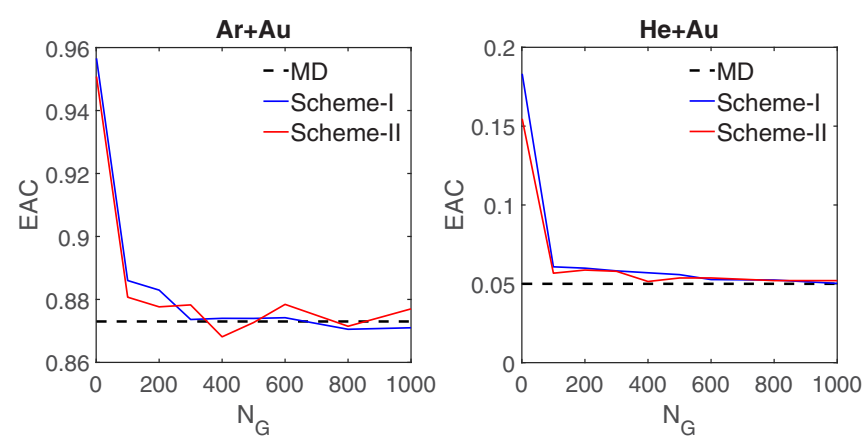

FIG. 6. Variation of the energy accommodation coefficient obtained from the MD simulation and the GM model using different numbers of Gaussian functions $N_{G}$. worthy to mention that, in the first approach, all the obtained velocity components by the GM model $\left(v_{x}^{\mathrm{GM}}, v_{y}^{\mathrm{GM}}, v_{z}^{\mathrm{GM}}\right)$ are Gaussian distributions. Therefore, using the reverse transfer function proposed in Ref. [32] and shown in Eq. (6) the normal velocity component is mapped back into the Rayleigh distribution, $v_{y}^{*, \mathrm{GM}}$, (step 5 in Scheme-I) to compare with the initial MD results. In Scheme-II the predicted normal velocity component by the GM model resembles a Rayleigh distribution. Therefore the obtained distributions can be used directly for the comparison purpose. Herein, the system with walls at the same temperature $\left(T_{b}=T_{t}=300 \mathrm{~K}\right)$, and without imposing any external velocity on the walls is used as a benchmark. The resulting velocity correlation distribution and PDF of the outgoing velocity for each molecular velocity component in the case of the He-Au and Ar-Au systems are shown in Figs. 3 and 4 , respectively.

As it is depicted in Fig. 3, for the He-Au system both Scheme-I and Scheme-II approaches are in a good agreement with MD simulation results. In the case of $\mathrm{Ar}-\mathrm{Au}$ (see Fig. 4) the only notable difference between MD, Scheme-I, and Schemea - II results is in the PDF of the normal velocity component (last column, second row).

For the Ar-Au system, the number of Gaussian functions $N_{G}$ employed in the GM model was increased to determine whether it can improve the predicted velocities by Scheme-II in the normal direction. In Fig. 5, the PDFs for the normal velocity component derived by Scheme-I based on $N_{G}=$ 100 (referred to as Scheme-I $I_{100}$ ), Scheme-II (referred to as Scheme- $\left.I I_{N_{G}}, 100 \leqslant N_{G} \leqslant 600\right)$, as well as MD results are represented. It is shown that by increasing $N_{G}$ the results obtained by Scheme-II converge towards the results obtained by MD simulations and the Scheme-I model, and at $N_{G}=600$ it is in excellent agreement with the other two methods.

In addition to the velocity correlations, the energy accommodation coefficient (EAC) was also computed to evaluate the performance of the GM model based on the introduced schemes for feeding the MD data into the model for training purposes. In each scheme $N_{G}$ was varied from $N_{G}=1$ to $N_{G}=1000$. In Fig. 6, it is seen that the variation in the EAC with increasing $N_{G}$ is rather smoother in the case of Scheme-I. In addition, for $N_{G}=600$ EAC computed by both GM models are in a perfect match with MD results.

From this analysis, it is deduced that generally Scheme-I has a better performance than Scheme-II. Therefore, to achieve high precision in the predicted velocity correlations and computed ACs, in the remaining part of this work Scheme-I based on $N_{G}=600$ is employed for training the GM model. The training of the model for each case takes within 10 to 12 minutes on a regular laptop computer.

\section{B. Performance evaluation of GM model}

In the next step, the performance of the GM model in comparison with the CLL model, which is one of the most used stochastic GSI models, and MD simulations results is studied. The comparison was carried out using the velocity correlation between impinging and outgoing velocities, and the PDF of the outgoing velocities in each spatial direction. Besides that, based on the predicted velocities via each of the GSI models, various accommodation coefficients including the tangential 

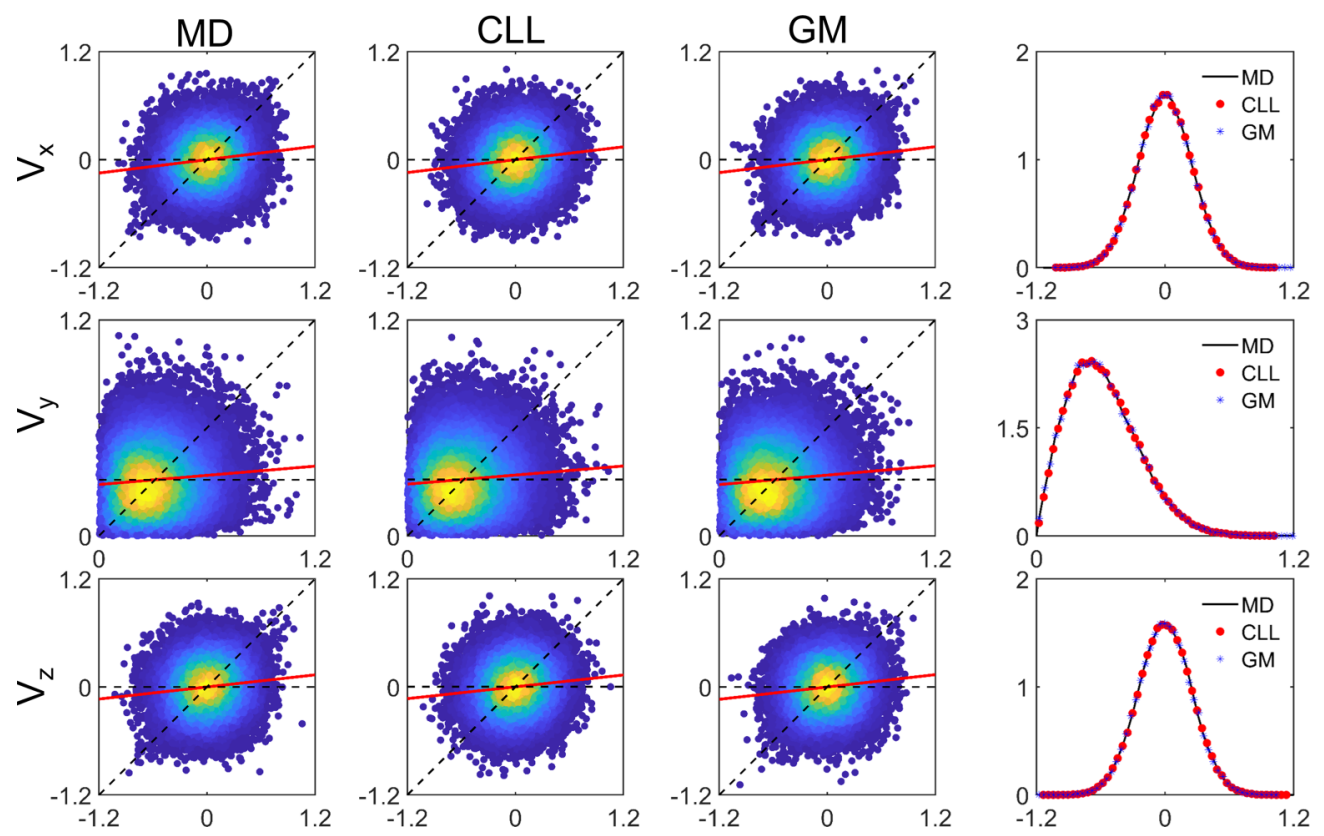

FIG. 7. Velocity correlations of impinging ( $x$ axis) and reflected ( $y$ axis) velocity components in $\mathrm{nm} / \mathrm{ps}$ for $\mathrm{Ar}-\mathrm{Au}$ system $\left(T_{b}=300 \mathrm{~K}\right.$, $T_{t}=300 \mathrm{~K}, S_{w}=0$ ). The dashed horizontal and diagonal lines indicate fully diffusive and specular conditions, respectively. Red lines indicate the least-square linear fit of the data. In the last column the corresponding probability density function for the reflecting particles is shown.

momentum accommodation coefficients in the both $z$ and $z$ directions $\left(\alpha_{x}, \alpha_{z}\right)$, the normal energy accommodation coefficient $\left(\alpha_{N E}\right)$, and the energy accommodation coefficient $\left(\alpha_{E}\right)$ are computed and verified against the MD simulations results.

For both $\mathrm{Au}-\mathrm{Ar}$ and $\mathrm{Au}-\mathrm{He}$ systems, two kinds of physical problems are investigated. In the first case study, the temperature of both walls are set to $300 \mathrm{~K}$, and in the second case, the temperature of the bottom wall surface $T_{b}$ is set to $300 \mathrm{~K}$, and that of the top wall surface $T_{t}$ is set to $500 \mathrm{~K}$. Besides that, in both cases the impact of flow condition on the performance of the GSI models hwas also investigated. As it is represented in Fig. 1, to achieve this goal an external velocity is imposed to the bottom and top walls in the $-x$ and $x$ directions, respectively. The following speed ratios are
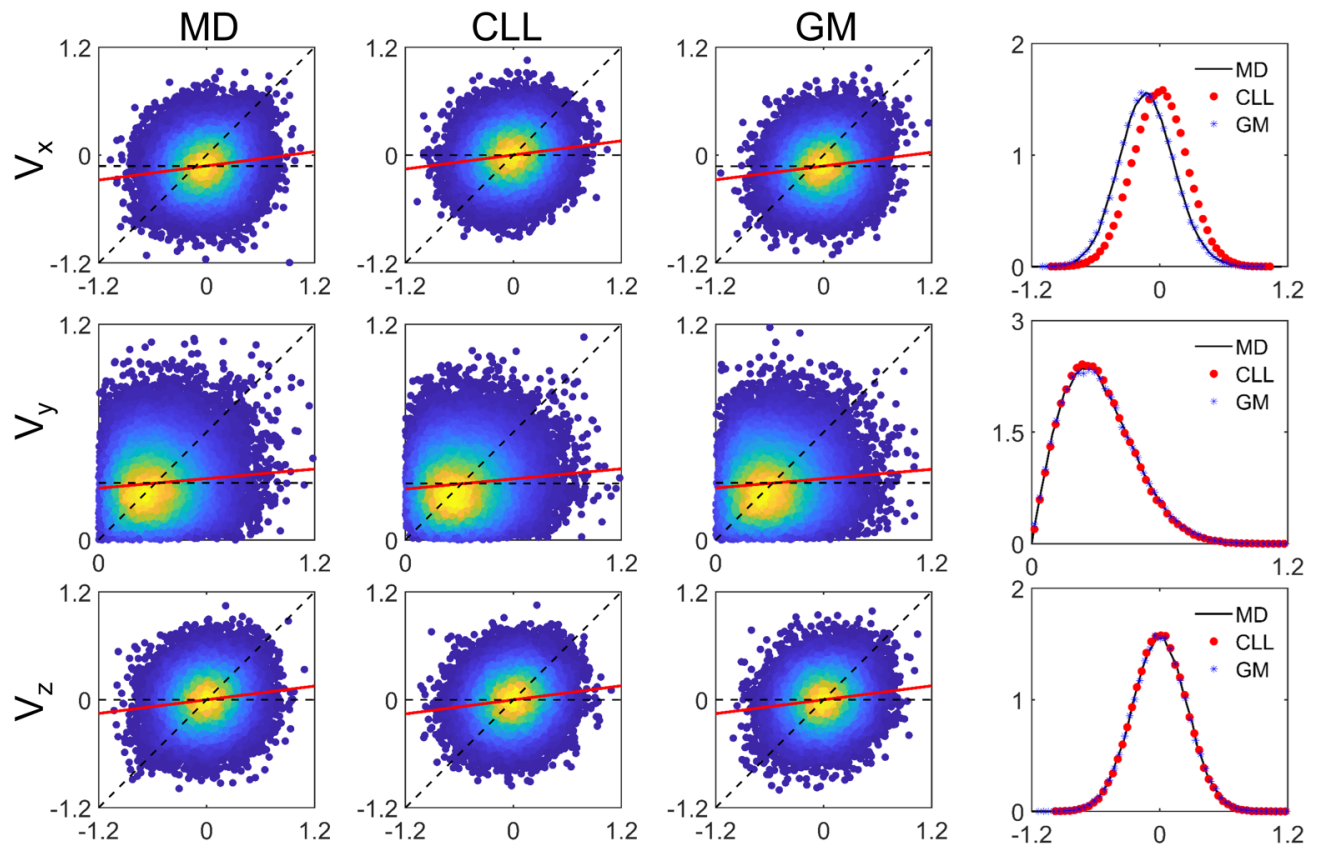

FIG. 8. Velocity correlations of impinging ( $x$ axis) and reflected ( $y$ axis) velocity components in $\mathrm{nm} / \mathrm{ps}$ for Ar-Au system at the bottom wall $\left(T_{b}=300 \mathrm{~K}, T_{t}=300 \mathrm{~K}, S_{w}=0.5\right)$. The dashed horizontal and diagonal lines indicate fully diffusive and specular conditions, respectively. Red lines indicate the least-square linear fit of the data. In the last column the corresponding probability density function for the reflecting particles is shown. 

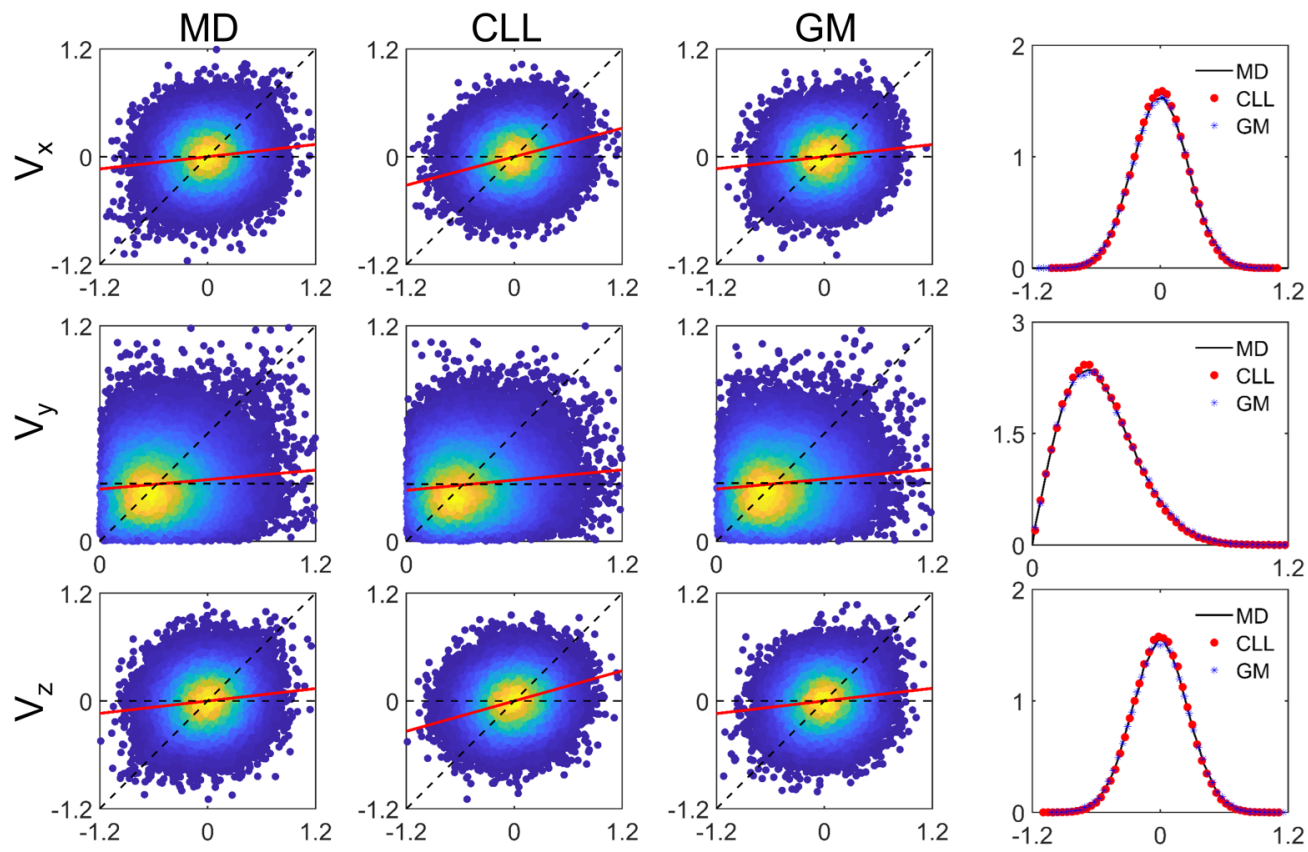

FIG. 9. Velocity correlations of impinging ( $x$ axis) and reflected ( $y$ axis) velocity components in $\mathrm{nm} / \mathrm{ps}$ for Ar-Au system at the bottom wall $\left(T_{b}=300 \mathrm{~K}, T_{t}=500 \mathrm{~K}, S_{w}=0\right)$. The dashed horizontal and diagonal lines indicate fully diffusive and specular conditions, respectively. Red lines indicate the least-square linear fit of the data. In the last column the corresponding probability density function for the reflecting particles is shown.

considered in the simulations: $S_{w}=0.25$ and $S_{w}=0.5$. For each system the most significant results are discussed in the main text and the remaining ones for Ar-Au system can be found in Appendix B (Figs. 16-19), and for He-Au can be found in Appendix C (Figs. 20-25).
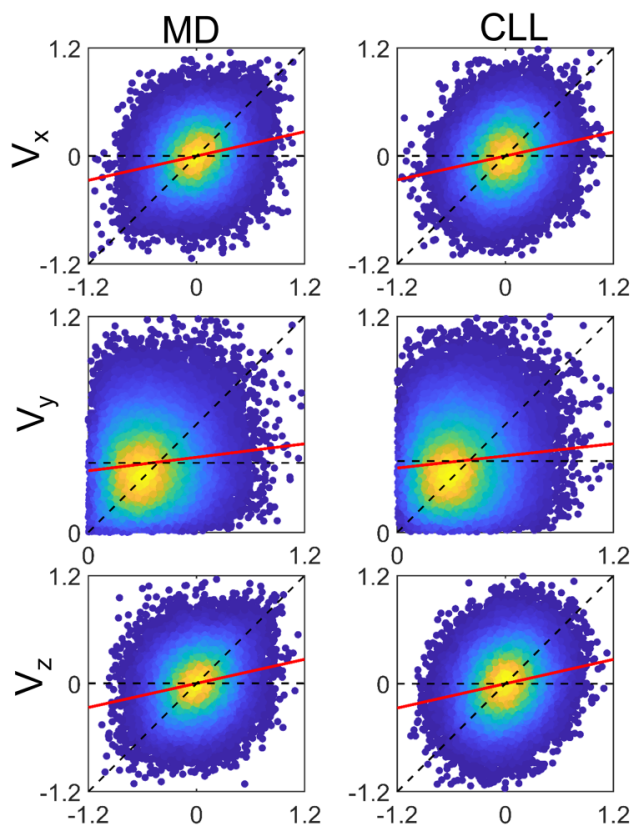

Normally, to derive a machine-learning-based model for a system, two different sets of data are required. The first set, called the training set, is used to derive the model parameters, and the second set, called the test set, is utilized to validate the model. In this study, before gathering the collisional data for computing accommodation coefficients (ACs) or training

FIG. 10. Velocity correlations of impinging ( $x$ axis) and reflected ( $y$ axis) velocity components in $\mathrm{nm} / \mathrm{ps}$ for Ar-Au system at the top wall $\left(T_{b}=300 \mathrm{~K}, T_{h}=500 \mathrm{~K}, S_{w}=0\right.$ ). The dashed horizontal and diagonal lines indicate fully diffusive and specular conditions, respectively. Red lines indicate the least-square linear fit of the data. In the last column the corresponding probability density function for the reflecting particles is shown. 

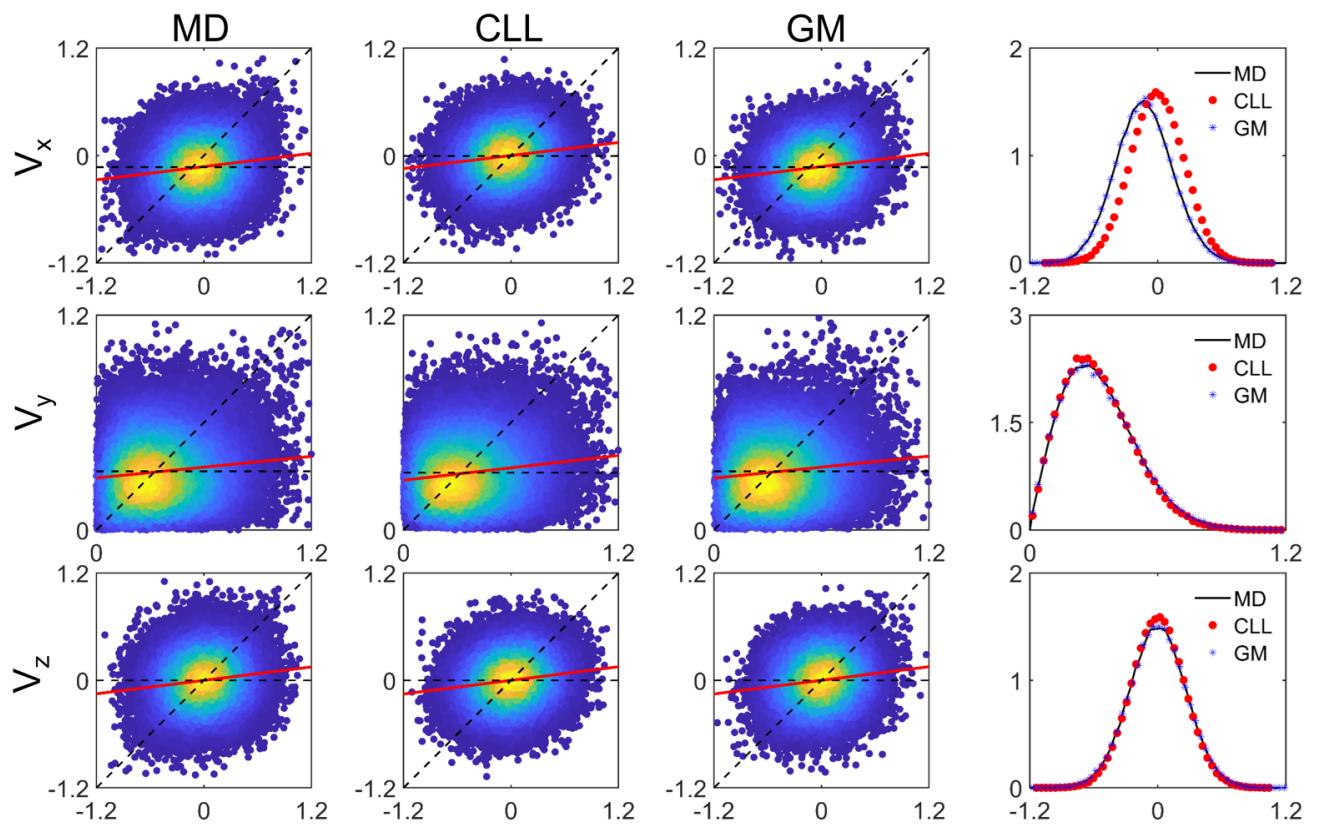

FIG. 11. Velocity correlations of impinging ( $x$ axis) and reflected ( $y$ axis) velocity components in $\mathrm{nm} / \mathrm{ps}$ for Ar-Au system at the bottom wall $\left(T_{b}=300 \mathrm{~K}, T_{t}=500 \mathrm{~K}, S_{w}=0.5\right)$. The dashed horizontal and diagonal lines indicate fully diffusive and specular conditions, respectively. Red lines indicate the least-square linear fit of the data. In the last column the corresponding probability density function for the reflecting particles is shown.

the GM model, it was ensured that the system has reached equilibrium conditions. Therefore, for each MD setup, characterized by a unique set of walls temperatures and velocities, there will not be any noticeable difference in collisional data obtained from different MD simulations. To prove this, for a specific MD setup, two independent MD simulations were conducted. One used as the training set $\left(\mathrm{MD}_{\text {train }}\right)$, and the other one used as the test set $\left(\mathrm{MD}_{\text {test }}\right)$. For each setup, using a different seed number in the Gaussian velocity generator, a different ensemble of initial velocities was created for the gas atoms. The velocity correlations between impinging and outgoing velocities, the PDF of the outgoing velocities, and the computed ACs are represented in the Appendix A (Fig. 15 and Table VI). It was shown that the obtained results from the $\mathrm{MD}_{\text {train }}, \mathrm{MD}_{\text {test }}$, and the GM model are in perfect match with each other.
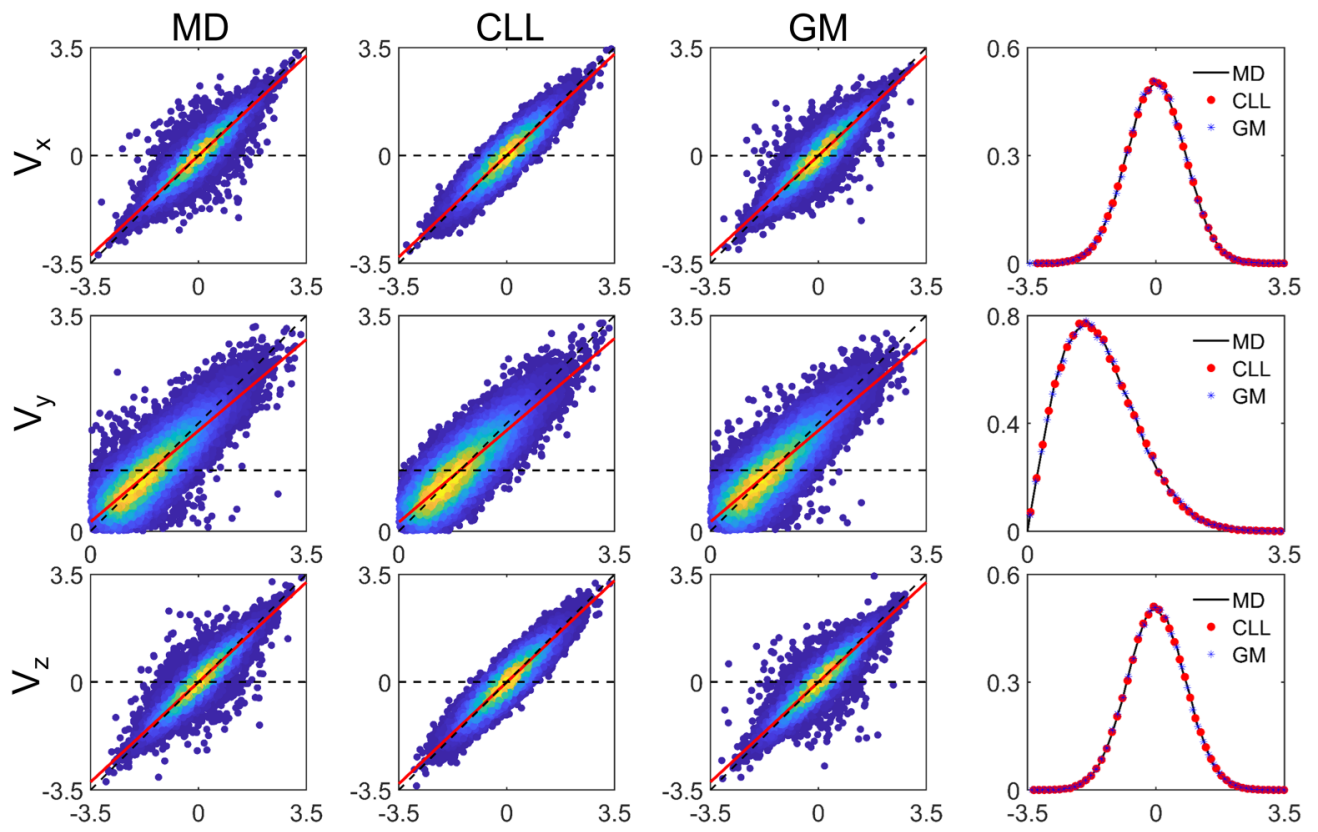

FIG. 12. Velocity correlations of impinging ( $x$ axis) and reflected ( $y$ axis) velocity components in $\mathrm{nm} / \mathrm{ps}$ for He-Au system $\left(T_{b}=300 \mathrm{~K}\right.$, $T_{t}=300 \mathrm{~K}, S_{w}=0$ ). The dashed horizontal and diagonal lines indicate fully diffusive and specular conditions, respectively. Red lines indicate the least-square linear fit of the data. In the last column the corresponding probability density function for the reflecting particles is shown. 

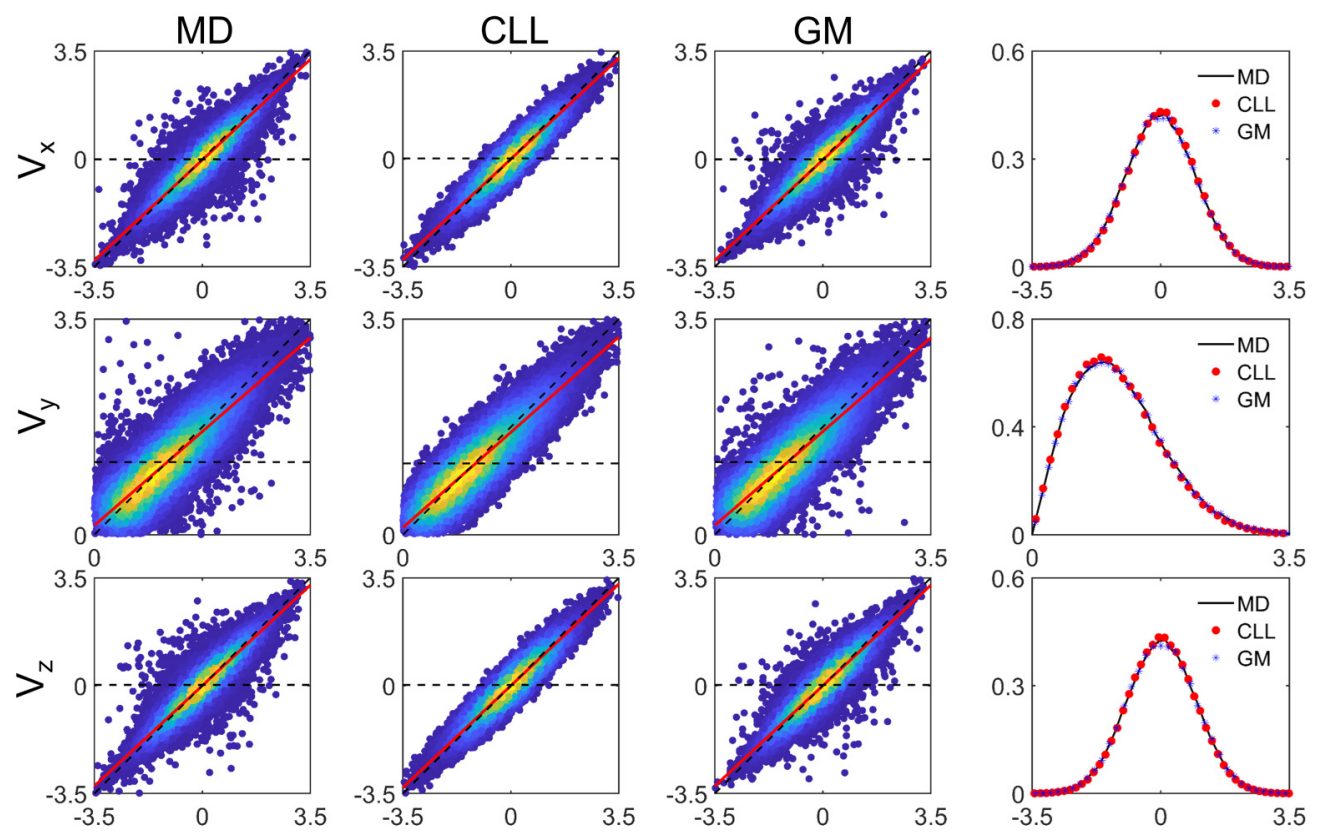

FIG. 13. Velocity correlations of impinging ( $x$ axis) and reflected ( $y$ axis) velocity components in $\mathrm{nm} / \mathrm{ps}$ for He-Au system $\left(T_{b}=300 \mathrm{~K}\right.$, $T_{t}=300 \mathrm{~K}, S_{w}=0.5$ ). The dashed horizontal and diagonal lines indicate fully diffusive and specular conditions, respectively. Red lines indicate the least-square linear fit of the data. In the last column the corresponding probability density function for the reflecting particles is shown.

\section{Ar-Au system}

Figure 7 shows the velocity correlations and the outgoing velocity PDFs in the case of a no-flow Ar-Au system, in which both walls have the same temperature $\left(T_{b}=T_{t}=300 \mathrm{~K}\right)$. It was observed when the gas and the both walls are in equilibrium state with each other, the predicted results by the
CLL and GM models are in a good agreement with the ones obtained by MD simulations.

In the next step, the flow velocity was added to the system by imposing a velocity to the walls in the the $x$ direction. As it is depicted in Fig. 8, which belongs to the case of $S_{w}=0.5$, there is a good agreement between the predicted PDFs in the $y$ and $z$ directions by both GSI models and the
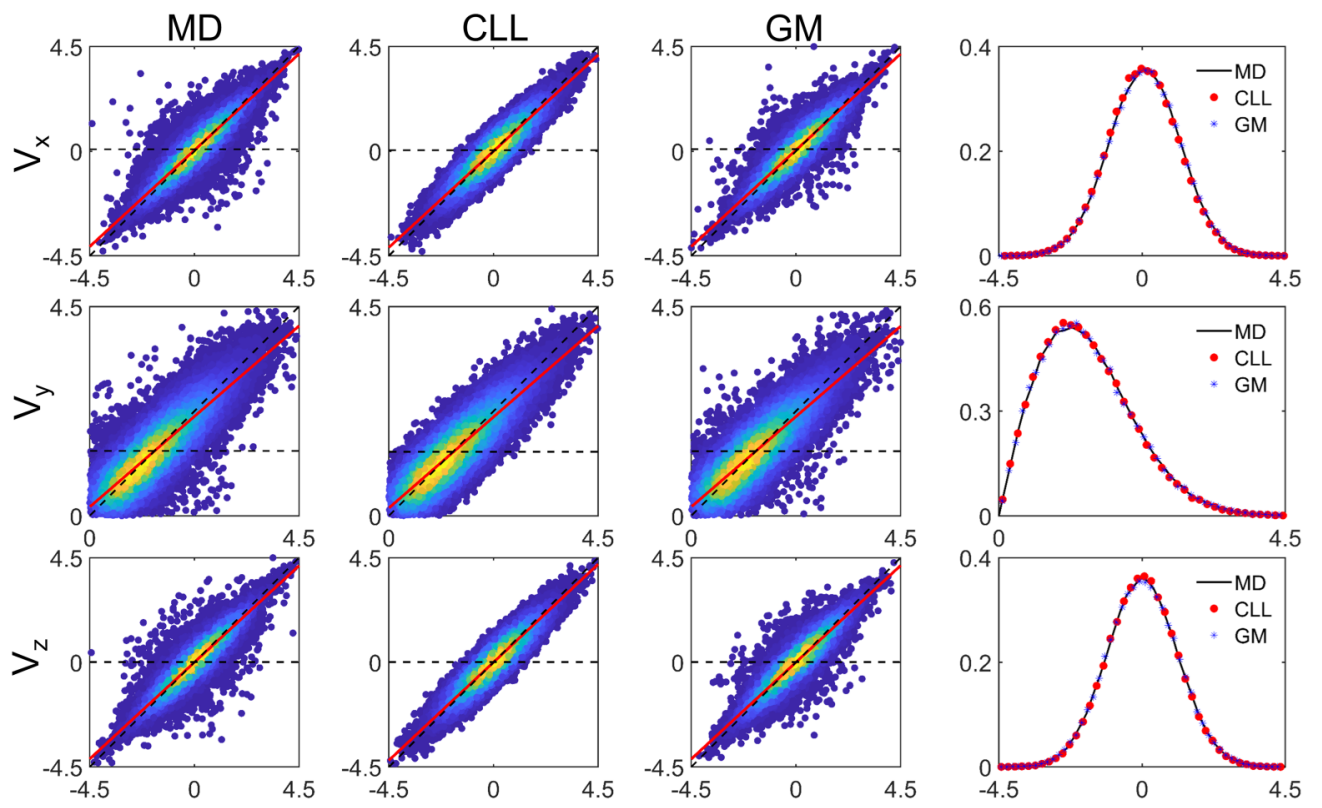

FIG. 14. Velocity correlations of impinging ( $x$ axis) and reflected ( $y$ axis) velocity components in $\mathrm{nm} / \mathrm{ps}$ for He-Au system at the top wall $\left(T_{b}=300 \mathrm{~K}, T_{t}=500 \mathrm{~K}, S_{w}=0.5\right.$ ). The dashed horizontal and diagonal lines indicate fully diffusive and specular conditions, respectively. Red lines indicate the least-square linear fit of the data. In the last column the corresponding probability density function for the reflecting particles is shown. 
MD simulations's results. On the contrary, in the $x$ direction, which is the direction of the imposed velocity, the results predicted by the CLL model deviate from the MD results while the results from the GM model are still in excellent agreement with the MD results. Herein, the mean value of the reflected velocities obtained from MD simulation and the GM model are 0.122 and $0.124 \mathrm{~nm} / \mathrm{ps}$, respectively. While the predicted mean value using the CLL model is $0.002 \mathrm{~nm} / \mathrm{ps}$. It is noteworthy to mention that for the system with $S_{w}=0.25$ the same kind of trend has been observed (see Appendix B). In this case, while the predicted mean velocities in the $x$ direction by MD simulation and the GM model are 0.0627 and $0.063 \mathrm{~nm} / \mathrm{ps}$, respectively, the value predicted by the CLL model is $0.001 \mathrm{~nm} / \mathrm{ps}$.

Figures 9 and 10 illustrate the resulting velocity correlations of impinging and reflected molecules after the collision with the bottom (cold) and the top (warm) walls in the case, in which there is temperature gradient between the walls $\left(T_{b}=300 \mathrm{~K}, T_{t}=500 \mathrm{~K}\right)$. It is seen that at the both walls the predicted results by the GM model are in perfect match with the MD simulations results. However, the results obtained by the CLL model for the tangential velocity components $\left(v_{x}, v_{z}\right)$ at both walls slightly deviated from the MD results at the peak of velocity PDFs.

The velocity distributions at the bottom wall for the system with temperature gradient between the walls and flow condition with $S_{w}=0.5$ are represented in Fig. 11. Similar to the earlier cases, the predicted results from the GM model have a very good agreement with MD simulations, while a mismatch is observed between results obtained by MD simulations and the CLL model in the tangential directions. This mismatch is more evident in the direction of imposed velocity (i.e., the $x$ direction).

For the Ar-Au system, the computed ACs in the case of systems with the walls at the same and different temperatures are reported in Tables II and III, respectively. First of all, by going through the results in the aforementioned tables it can be understood that imposing the external velocity does not have a significant impact on the obtained ACs on the walls. Such behavior was also reported in Ref. [48], where a gas kinetic model was employed to study the GSI in a Couette flow system. Besides that, in the case of the system with the walls at different temperatures (see Table III) all the ACs computed on the top wall are lower than the ones computed on the bottom wall. This behavior is due to the higher surface temperature at the top wall and was also previously reported [25]. Considering the directional accommodation coefficients including $\alpha_{x}, \alpha_{z}, \alpha_{N E}$, it was observed that the results obtained by both GSI models are in good agreement with the MD simulation results. However, it is found that $\alpha_{E}$ obtained by the CLL model is slightly higher that the MD results, whereas the results obtained by the GM model is always in perfect match with the MD ones.

\section{He-Au system}

The same sort of assessment was also carried out in the case of the He-Au system. The velocity distributions for the system, in which both walls have the same temperature $\left(T_{b}=\right.$
TABLE II. Tangential momentum $\left(\alpha_{x}, \alpha_{z}\right)$, normal energy $\left(\alpha_{\mathrm{NE}}\right)$, and energy $\left(\alpha_{E}\right)$ accommodation coefficients for two parallel walls $\mathrm{Ar}-\mathrm{Au}$ system with the walls at the same temperature at various speed ratios $\left(S_{w}\right)$, computed using different stochastic approaches: CLL and GM models and MD simulations.

\begin{tabular}{lcclll}
\hline \hline$S_{w}$ & Model & $\alpha_{x}$ & $\alpha_{z}$ & $\alpha_{\mathrm{NE}}$ & $\alpha_{E}$ \\
\hline \multirow{4}{*}{0} & MD & 0.876 & 0.888 & 0.91 & 0.873 \\
& CLL & 0.877 & 0.886 & 0.91 & 0.947 \\
& GM & 0.879 & 0.888 & 0.909 & 0.884 \\
0.25 & MD & 0.879 & 0.88 & 0.916 & 0.878 \\
& CLL & 0.873 & 0.878 & 0.914 & 0.95 \\
& GM & 0.879 & 0.877 & 0.921 & 0.888 \\
0.5 & MD & 0.868 & 0.873 & 0.903 & 0.87 \\
& CLL & 0.864 & 0.88 & 0.901 & 0.94 \\
& GM & 0.872 & 0.871 & 0.911 & 0.891 \\
\hline \hline
\end{tabular}

$T_{t}=300 \mathrm{~K}$ ), without and with imposing the external velocity $\left(S_{w}=0.5\right)$ are shown in Figs. 12 and 13, respectively. Comparing the velocity correlations obtained by the molecular simulations and the GSI models, the most significant observed difference is that the results from the CLL model are narrower than those computed by the MD simulations and GM model. Furthermore, inducing wall velocity (see Fig. 13) does not have any noticeable influence on the system behavior, and as it is also illustrated in the aforementioned figures, the velocity PDFs obtained by both GSI models are in accordance with

TABLE III. Tangential momentum $\left(\alpha_{x}, \alpha_{z}\right)$, normal energy $\left(\alpha_{\mathrm{NE}}\right)$, and energy $\left(\alpha_{E}\right)$ accommodation coefficients for two parallel walls $\mathrm{Ar}-\mathrm{Au}$ system with a temperature gradient between the walls at various speed ratios $\left(S_{w}\right)$, computed using different stochastic approaches: CLL and GM models and MD simulations. B: bottom wall; T: top wall.

\begin{tabular}{|c|c|c|c|c|c|c|}
\hline$S_{w}$ & Model & Wall & $\alpha_{x}$ & $\alpha_{z}$ & $\alpha_{\mathrm{NE}}$ & $\alpha_{E}$ \\
\hline \multirow{6}{*}{0} & \multirow[t]{2}{*}{ MD } & B & 0.886 & 0.884 & 0.91 & 0.873 \\
\hline & & $\mathrm{T}$ & 0.775 & 0.777 & 0.856 & 0.792 \\
\hline & \multirow[t]{2}{*}{ CLL } & B & 0.884 & 0.884 & 0.908 & 0.943 \\
\hline & & $\mathrm{T}$ & 0.778 & 0.777 & 0.855 & 0.907 \\
\hline & \multirow[t]{2}{*}{ GM } & B & 0.887 & 0.882 & 0.908 & 0.882 \\
\hline & & $\mathrm{T}$ & 0.767 & 0.772 & 0.871 & 0.81 \\
\hline & \multirow[t]{2}{*}{ MD } & B & 0.875 & 0.876 & 0.898 & 0.865 \\
\hline & & $\mathrm{T}$ & 0.771 & 0.769 & 0.851 & 0.778 \\
\hline \multirow[t]{6}{*}{0.25} & \multirow[t]{2}{*}{ CLL } & B & 0.877 & 0.881 & 0.899 & 0.937 \\
\hline & & $\mathrm{T}$ & 0.772 & 0.766 & 0.848 & 0.901 \\
\hline & \multirow[t]{2}{*}{ GM } & B & 0.877 & 0.88 & 0.903 & 0.883 \\
\hline & & $\mathrm{T}$ & 0.772 & 0.773 & 0.851 & 0.79 \\
\hline & \multirow[t]{2}{*}{ MD } & B & 0.876 & 0.875 & 0.897 & 0.869 \\
\hline & & $\mathrm{T}$ & 0.76 & 0.761 & 0.844 & 0.773 \\
\hline \multirow[t]{4}{*}{0.5} & \multirow[t]{2}{*}{ CLL } & B & 0.875 & 0.875 & 0.898 & 0.94 \\
\hline & & $\mathrm{T}$ & 0.76 & 0.763 & 0.843 & 0.897 \\
\hline & \multirow[t]{2}{*}{ GM } & B & 0.877 & 0.873 & 0.897 & 0.877 \\
\hline & & $\mathrm{T}$ & 0.76 & 0.767 & 0.844 & 0.789 \\
\hline
\end{tabular}


TABLE IV. Tangential momentum $\left(\alpha_{x}, \alpha_{z}\right)$, normal energy $\left(\alpha_{\mathrm{NE}}\right)$, and energy $\left(\alpha_{E}\right)$ accommodation coefficients for two parallel walls $\mathrm{He}-\mathrm{Au}$ system with the walls at the same temperature at various speed ratios $\left(S_{w}\right)$, computed using different stochastic approaches: CLL and GM models and MD simulations.

\begin{tabular}{lcclll}
\hline \hline$S_{w}$ & Model & $\alpha_{x}$ & \multicolumn{1}{c}{$\alpha_{z}$} & $\alpha_{\mathrm{NE}}$ & \multicolumn{1}{c}{$\alpha_{E}$} \\
\hline \multirow{4}{*}{0} & MD & 0.073 & 0.073 & 0.132 & 0.05 \\
& CLL & 0.073 & 0.073 & 0.131 & 0.137 \\
& GM & 0.074 & 0.073 & 0.13 & 0.052 \\
0.25 & MD & 0.069 & 0.0 .072 & 0.126 & 0.042 \\
& CLL & 0.069 & 0.073 & 0.125 & 0.131 \\
& GM & 0.0 .07 & 0.0 .07 & 0.131 & 0.046 \\
0.5 & MD & 0.067 & 0.068 & 0.118 & 0.041 \\
& CLL & 0.067 & 0.068 & 0.115 & 0.123 \\
& GM & 0.068 & 0.068 & 0.115 & 0.043 \\
\hline \hline
\end{tabular}

the MD results. The resulting velocity distributions at the top wall of a system, in which two walls are kept at different temperatures $\left(T_{b}=T_{t}=500 \mathrm{~K}\right)$, and enforced by an external wall velocity $\left(S_{w}=0.5\right)$ are depicted in Fig. 14 . Similar to the previous system with the same wall temperatures, it is seen that also in this system, the only considerable difference between the obtained results from the GSI models and MD simulation is that, in terms of sparsity, the velocity correlation based on the GM model and MD results resemble each other more. It is deduced that in comparison with the Ar-Au system,

TABLE V. Tangential momentum $\left(\alpha_{x}, \alpha_{z}\right)$, normal energy $\left(\alpha_{\mathrm{NE}}\right)$, and energy $\left(\alpha_{E}\right)$ accommodation coefficients for two parallel walls $\mathrm{He}-\mathrm{Au}$ system with a temperature gradient between the walls at various speed ratios $\left(S_{w}\right)$, computed using different stochastic approaches: CLL and GM models and MD simulations. B: bottom wall; $\mathrm{T}$ : top wall.

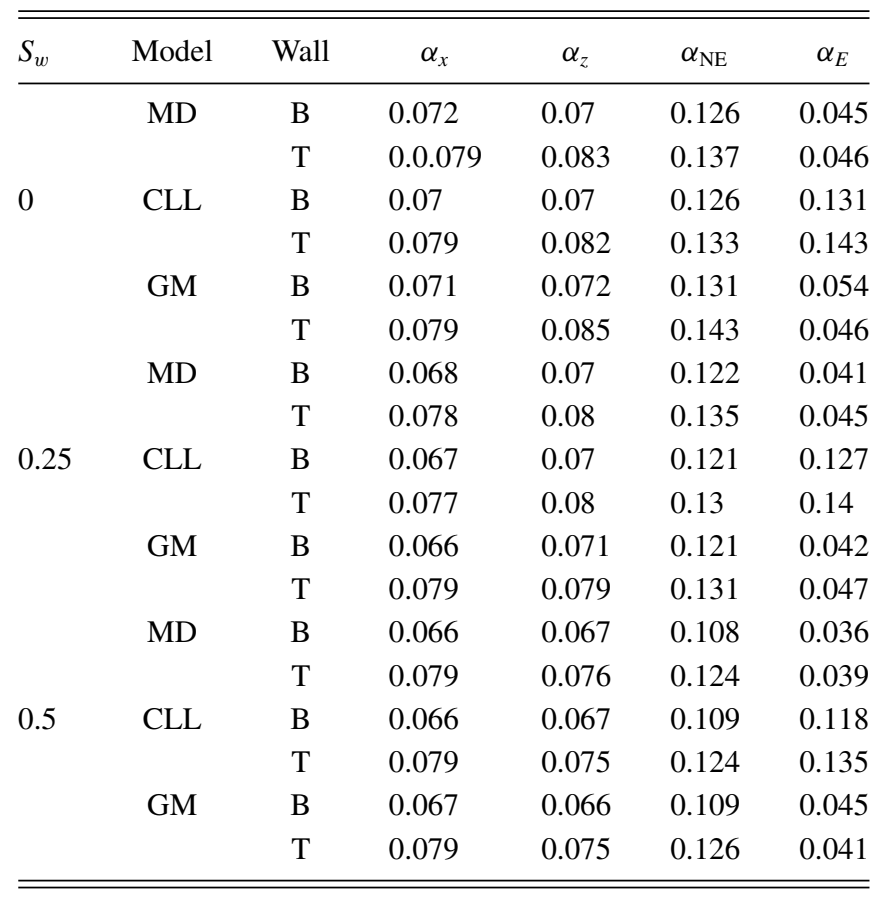

in the current case introducing various kinds of perturbations, such as the temperature difference between the walls or imposing an external shear flow on the system does not affect the results obtained by MD simulation and GSI models, significantly. This behavior is mainly caused by the essence of relatively weak interaction strength between $\mathrm{He}$ gas and the $\mathrm{Au}$ surface, which leads to rather perfectly elastic reflection of the He atoms from the Au surface. Tables IV and V present various ACs obtained from the employed GSI models, as well as MD simulations for the $\mathrm{He}-\mathrm{Au}$ system without and with a temperature gradient between the two walls, respectively. First of all, considering only the MD-obtained results it is observed that the majority of ACs for He-Au system are one order of magnitude smaller than those obtained for Ar-Au system for the same system condition. This issue, which was also reported in Ref. [32], confirms further the presence of the weaker interaction potential at the $\mathrm{He}-\mathrm{Au}$ interface in the comparison with the Ar-Au interface. Furthermore, it was noticed while the calculated directional ACs based on the predicted velocities by both CLL and GM models are in a good agreement with the MD results, the CLL model considerably overpredicts the value of $\alpha_{E}$. However, the GM model shows high degree of accuracy in all the computed ACs.

\section{CONCLUSION}

In this study, the GM model, an unsupervised machine learning technique, is employed to investigate the interfacial interactions between two noble gases (Ar,He) with Au solid surface. The main advantage of the scattering kernel constructed based on the GM model over the existing stochastic empirical scattering kernels is that its capability is not restricted by the finite number of adjustable parameters, which are required to be known in advance. The GM model uses a superposition of multivariate normal distributions to derive the probability density of a high-dimensional data space. Here the whole collisional data obtained from MD simulation is used for training the model. This guarantees that the obtained model preserves all the important physics in the MD collisional data. The performance of the model in the case of a thermally nonequilibrium, as well as a system with moving walls, which are encountered in various applications involving dilute gas, has been evaluated. The assessment was performed in terms of both statistical and physical characteristics of the system, such as the velocity correlation between incoming and outgoing velocities and the PDF of the outgoing velocities, as well as different accommodation coefficients obtained using the predicted postcollisional velocities by the model. Comparing the results obtained from the GM and CLL models, with the MD results showed the excellent performance of the GM model. Therefore, in the case of monoatomic gases, this model, as a generalized form of the collisional model, can be coupled to the coarse-grained simulation techniques, such as DSMC or LBM, and removes the need for the computationally demanding MD simulations. To derive such a generalized scattering kernel, a more extended data set including wider range of the wall temperatures and velocities, as well as different values for gas density is required, and this is in our plan for future studies. It is noteworthy to mention that the 
high accuracy of the predicted postcollisional velocities by the GM model makes it also a promising candidate for computing various important physical parameters at the gas-wall interface, such as ACs, shear stress, and thermal conductance in the system with highly nonequilibrium gas flow.

\section{ACKNOWLEDGMENTS}

This work is part of the research program RareTrans with Project No. HTSM-15376, which is (partly) financed by the Netherlands Organization for Scientific Research (NWO).
TABLE VI. Tangential momentum $\left(\alpha_{x}, \alpha_{z}\right)$, normal energy $\left(\alpha_{\mathrm{NE}}\right)$, and energy $\left(\alpha_{E}\right)$ accommodation coefficients for two parallel walls $\mathrm{Ar}-\mathrm{Au}$ system at the bottom wall $\left(T_{b}=300 \mathrm{~K}, T_{t}=500 \mathrm{~K}\right.$, $S_{w}=0.5$ ), using MD training and test data sets, as well as the GM model.

\begin{tabular}{lcccc}
\hline \hline Model & $\alpha_{x}$ & $\alpha_{z}$ & $\alpha_{\mathrm{NE}}$ & $\alpha_{E}$ \\
\hline MD $_{\text {train }}$ & 0.876 & 0.875 & 0.897 & 0.869 \\
MD $_{\text {test }}$ & 0.874 & 0.876 & 0.895 & 0.866 \\
$\mathrm{GM}$ & 0.877 & 0.873 & 0.897 & 0.877 \\
\hline \hline
\end{tabular}

\section{APPENDIX A}

Comparison between velocity correlations, PDFs, and ACs obtained from training $\left(\mathrm{MD}_{\text {train }}\right)$, testing $\left(\mathrm{MD}_{\text {test }}\right)$ and the $\mathrm{GM}$ model for Ar-Au system.
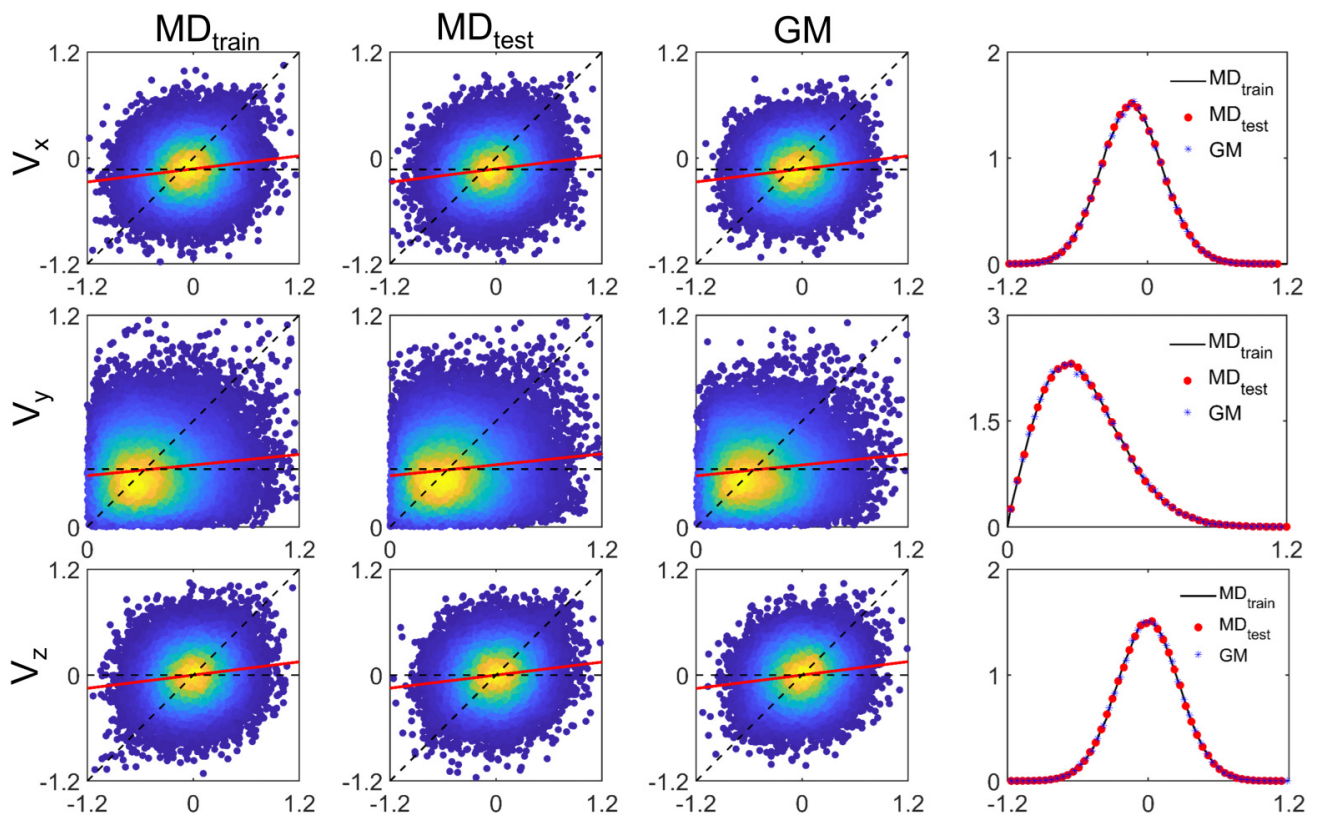

FIG. 15. Velocity correlations of impinging ( $x$ axis) and reflected ( $y$ axis) velocity components in $\mathrm{nm} / \mathrm{ps}$ for Ar-Au system at the bottom wall $\left(T_{b}=300 \mathrm{~K}, T_{t}=500 \mathrm{~K}, S_{w}=0.5\right)$. The dashed horizontal and diagonal lines indicate fully diffusive and specular conditions, respectively. Red lines indicate the least-square linear fit of the data. In the last column the corresponding probability density function for the reflecting particles is shown. 


\section{APPENDIX B}

Velocity correlations and PDFs for some of the Ar-Au case studies, which are not discussed in the main text.
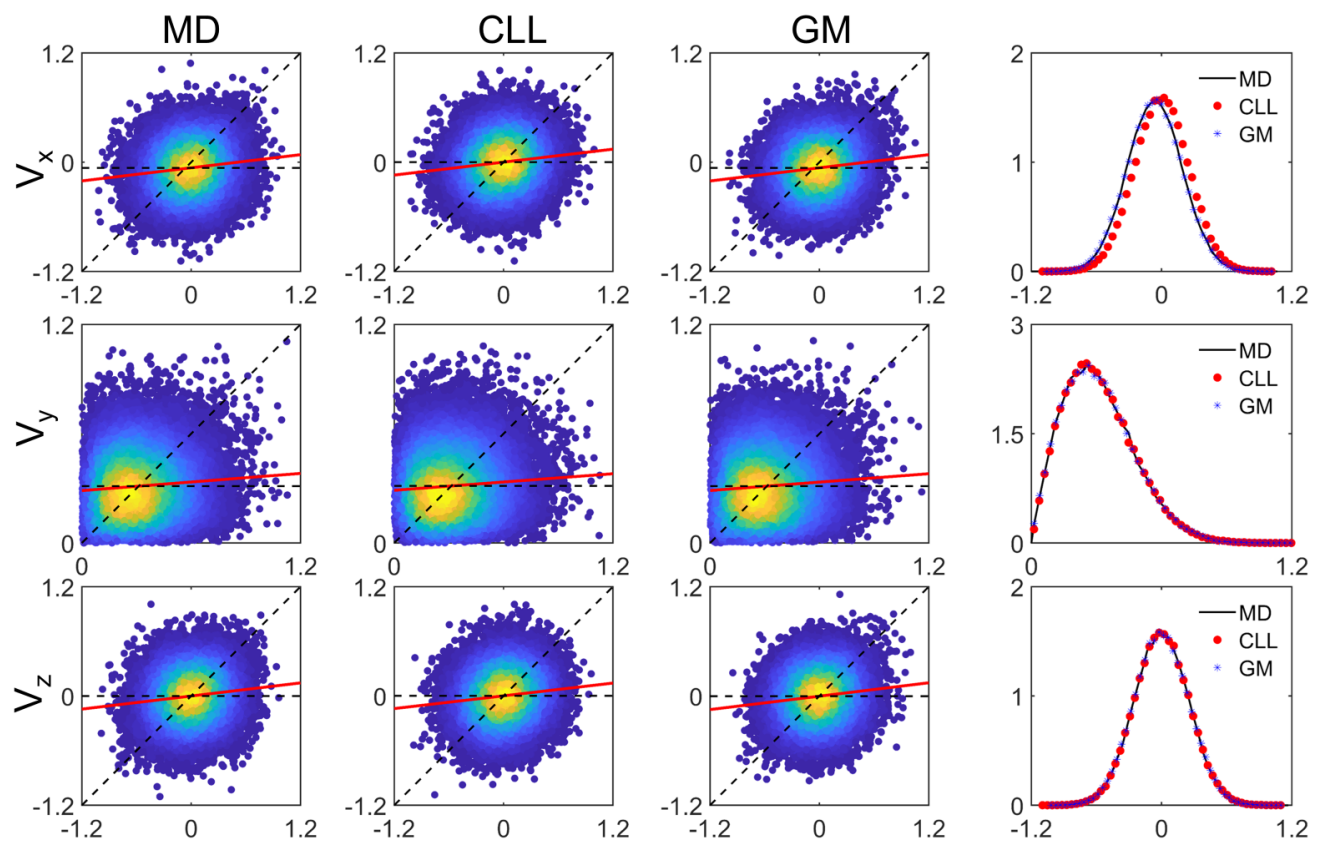

FIG. 16. Velocity correlations of impinging ( $x$ axis) and reflected ( $y$ axis) velocity components in $\mathrm{nm} / \mathrm{ps}$ for $\mathrm{Ar}-\mathrm{Au}$ system $\left(T_{b}=300 \mathrm{~K}\right.$, $T_{t}=300 \mathrm{~K}, S_{w}=0.25$ ). The dashed horizontal and diagonal lines indicate fully diffusive and specular conditions, respectively. Red lines indicate the least-square linear fit of the data. In the last column the corresponding probability density function for the reflecting particles is shown.
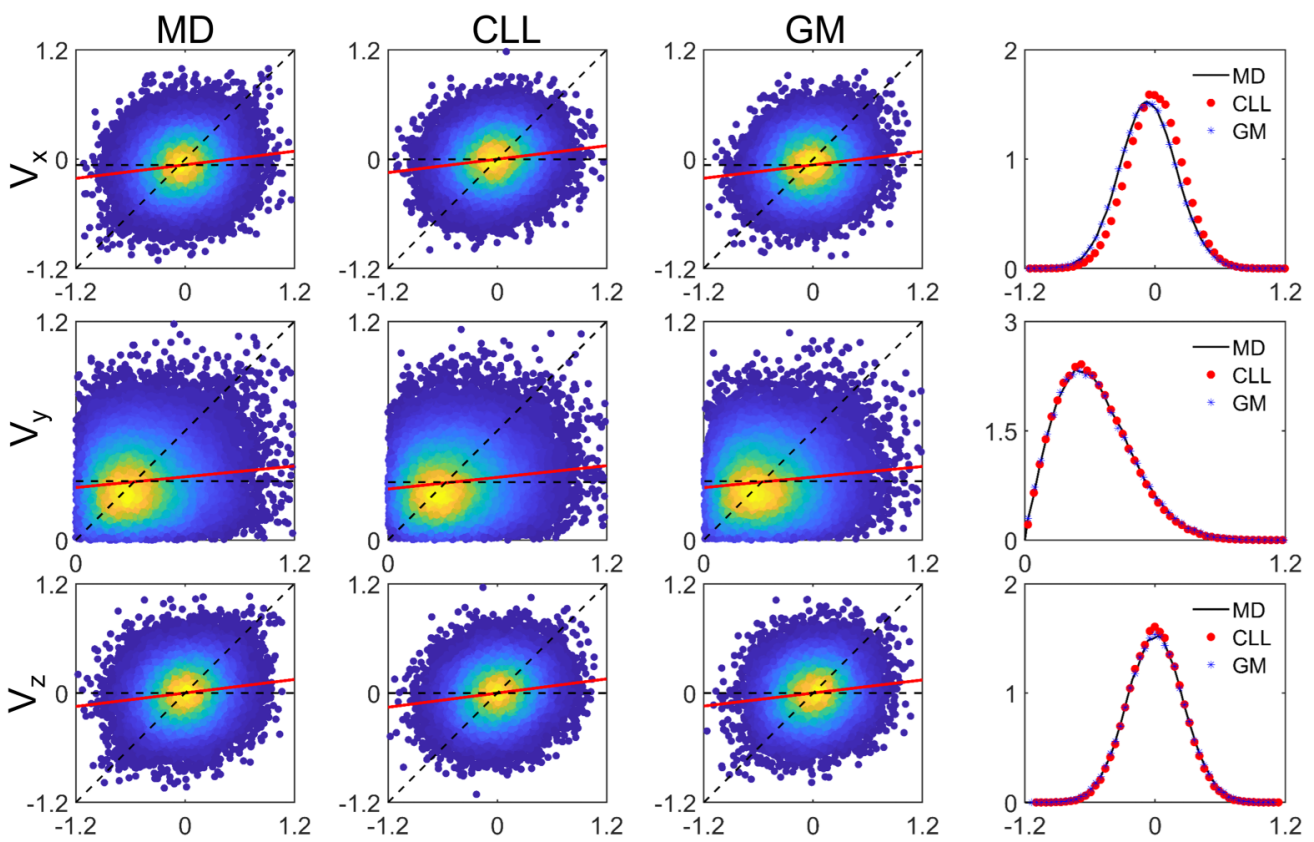

FIG. 17. Velocity correlations of impinging ( $x$ axis) and reflected ( $y$ axis) velocity components in $\mathrm{nm} / \mathrm{ps}$ for Ar-Au system at the bottom wall $\left(T_{b}=300 \mathrm{~K}, T_{t}=500 \mathrm{~K}, S_{w}=0.25\right)$. The dashed horizontal and diagonal lines indicate fully diffusive and specular conditions, respectively. Red lines indicate the least-square linear fit of the data. In the last column the corresponding probability density function for the reflecting particles is shown. 

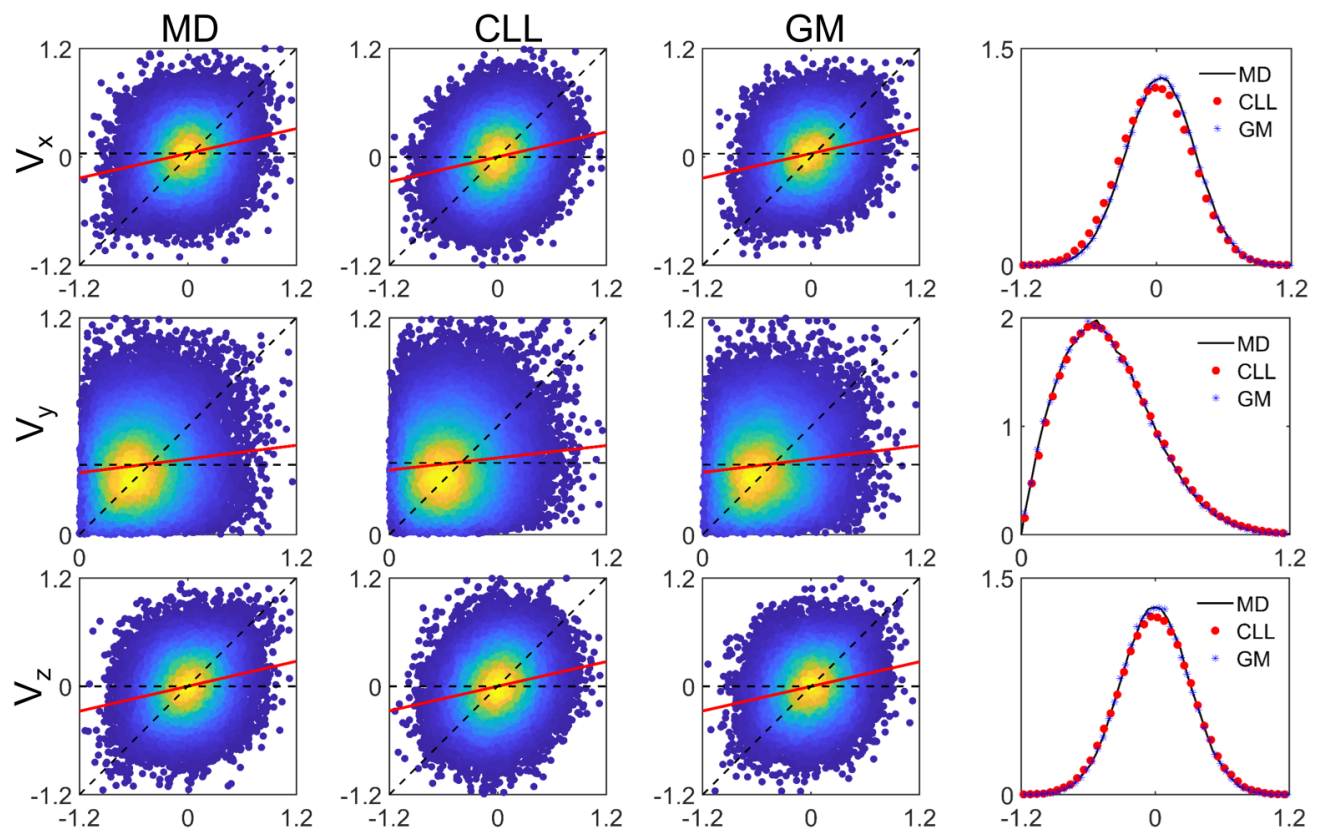

FIG. 18. Velocity correlations of impinging ( $x$ axis) and reflected ( $y$ axis) velocity components in $\mathrm{nm} / \mathrm{ps}$ for Ar-Au system at the top wall $\left(T_{b}=300 \mathrm{~K}, T_{t}=500 \mathrm{~K}, S_{w}=0.25\right.$ ). The dashed horizontal and diagonal lines indicate fully diffusive and specular conditions, respectively. Red lines indicate the least-square linear fit of the data. In the last column the corresponding probability density function for the reflecting particles is shown.
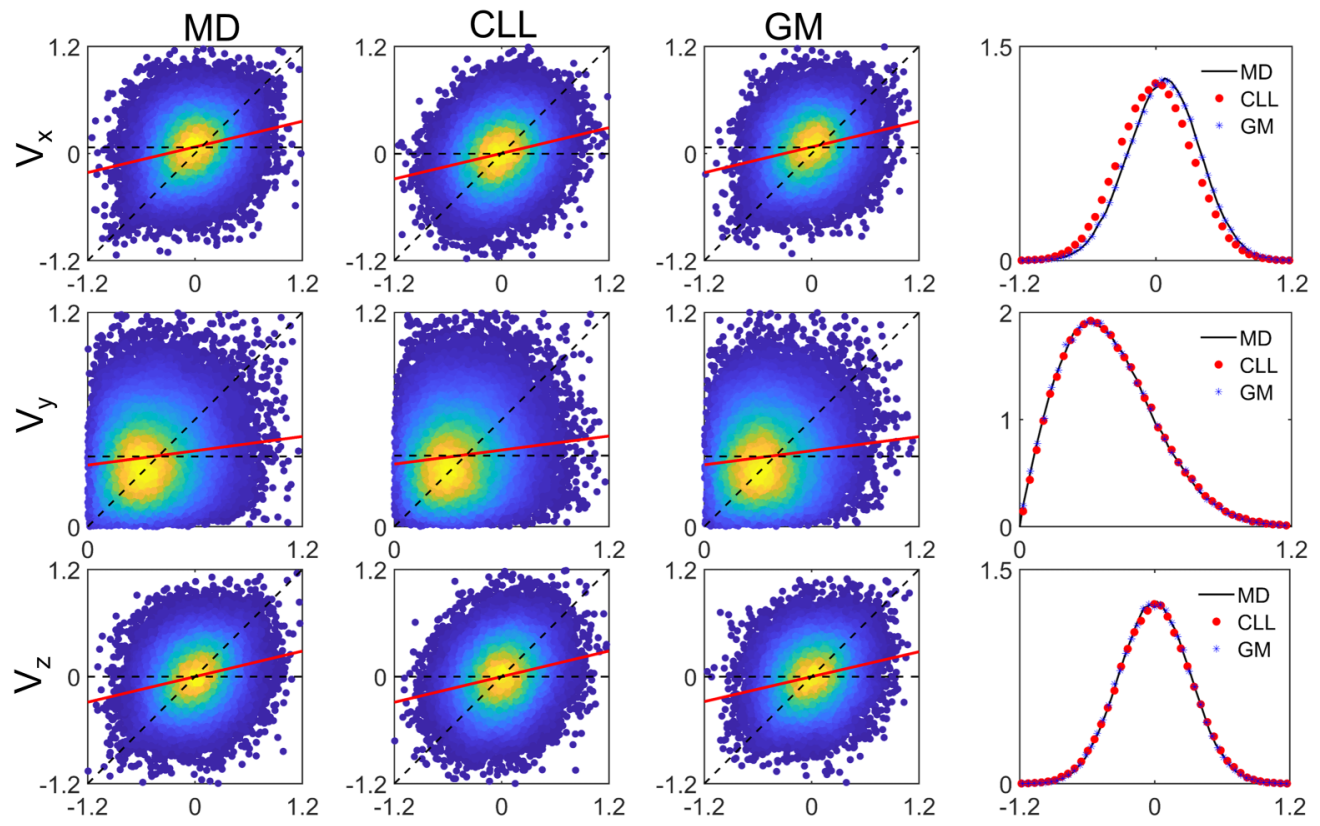

FIG. 19. Velocity correlations of impinging ( $x$ axis) and reflected ( $y$ axis) velocity components in $\mathrm{nm} / \mathrm{ps}$ for Ar-Au system at the top wall $\left(T_{b}=300 \mathrm{~K}, T_{t}=500 \mathrm{~K}, S_{w}=0.5\right.$ ). The dashed horizontal and diagonal lines indicate fully diffusive and specular conditions, respectively. Red lines indicate the least-square linear fit of the data. In the last column the corresponding probability density function for the reflecting particles is shown. 


\section{APPENDIX C}

Velocity correlations and PDFs for some of the He-Au case studies, which are not discussed in the main text.
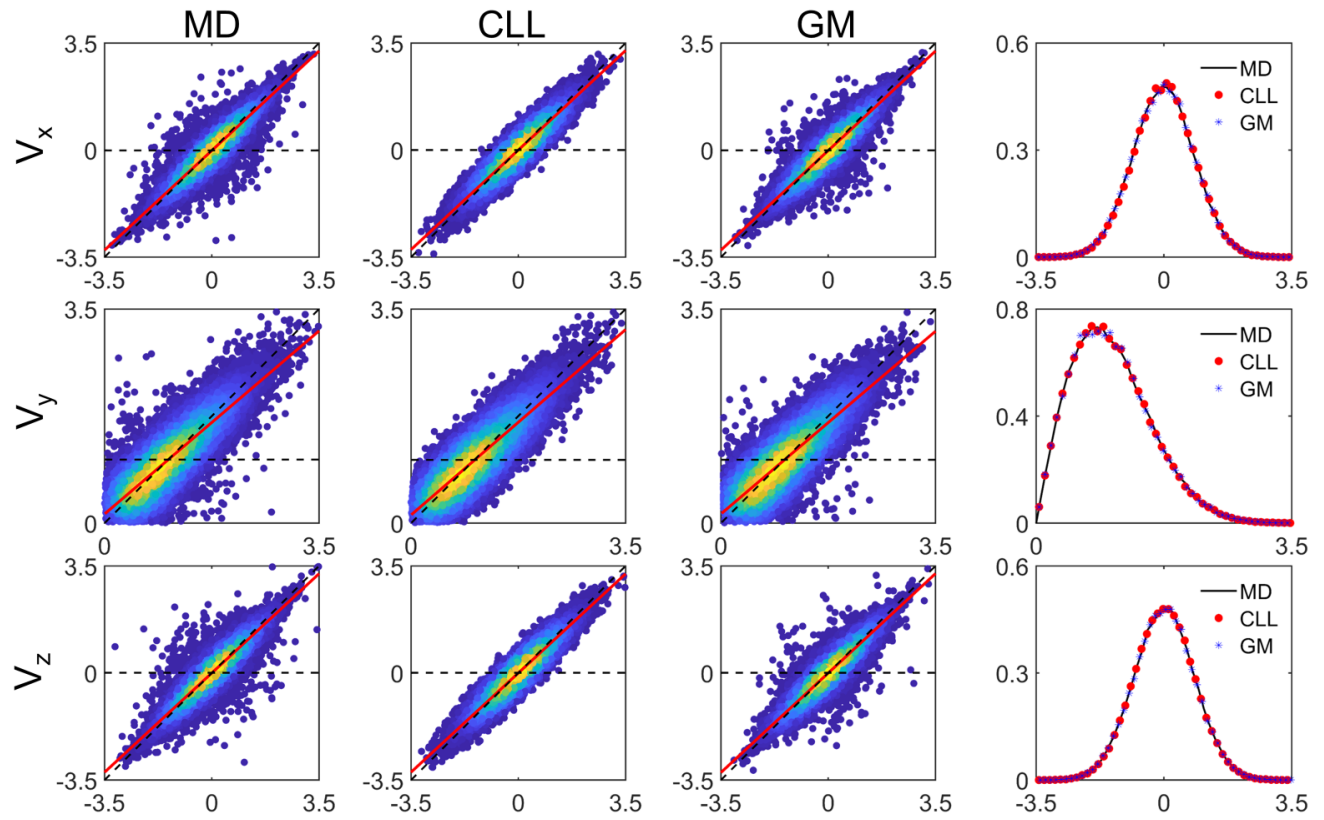

FIG. 20. Velocity correlations of impinging ( $x$ axis) and reflected ( $y$ axis) velocity components in $\mathrm{nm} / \mathrm{ps}$ for He-Au system $\left(T_{b}=300 \mathrm{~K}\right.$, $T_{t}=300 \mathrm{~K}, S_{w}=0.25$ ). The dashed horizontal and diagonal lines indicate fully diffusive and specular conditions, respectively. Red lines indicate the least-square linear fit of the data. In the last column the corresponding probability density function for the reflecting particles is shown.
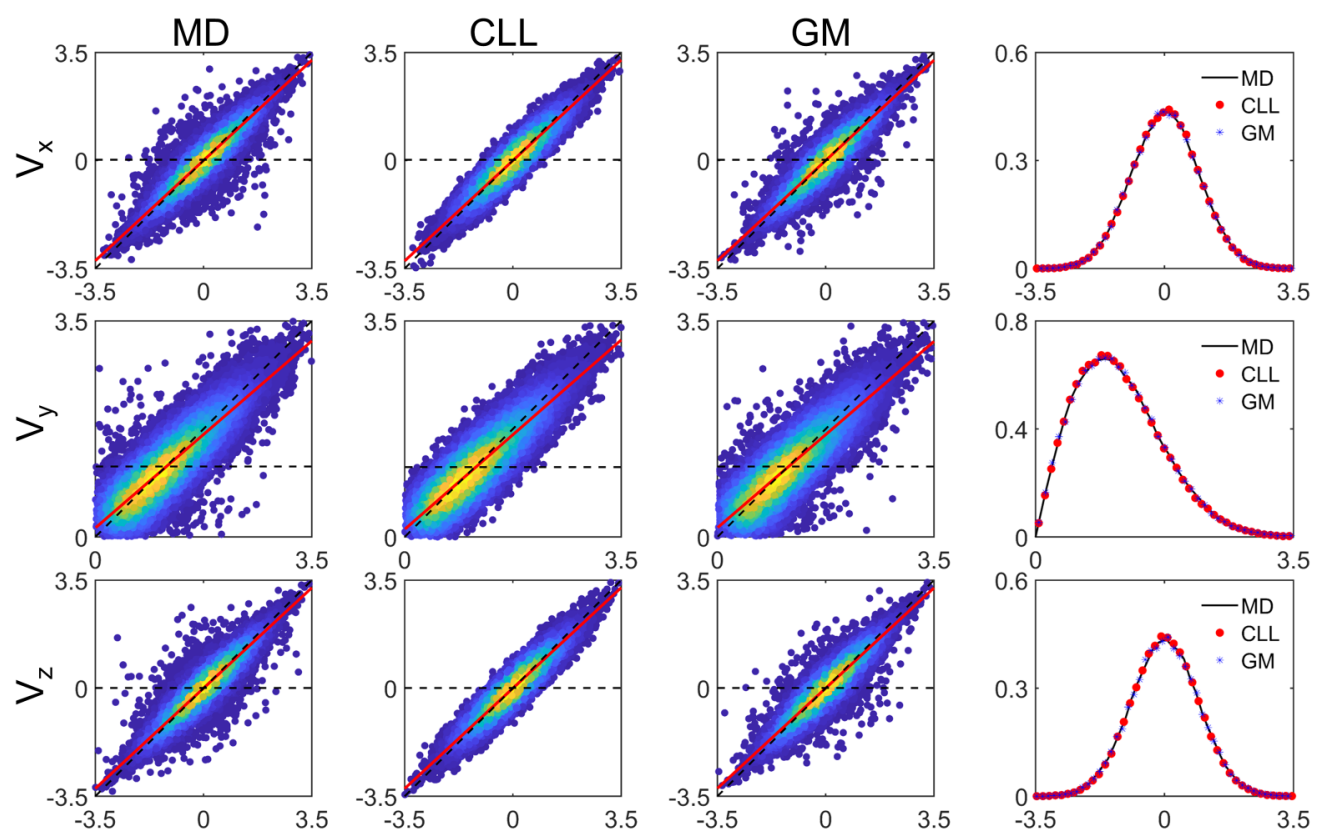

FIG. 21. Velocity correlations of impinging ( $x$ axis) and reflected ( $y$ axis) velocity components in $\mathrm{nm} / \mathrm{ps}$ for He-Au system at the bottom wall $\left(T_{b}=300 \mathrm{~K}, T_{t}=500 \mathrm{~K}, S_{w}=0\right)$. The dashed horizontal and diagonal lines indicate fully diffusive and specular conditions, respectively. Red lines indicate the least-square linear fit of the data. In the last column the corresponding probability density function for the reflecting particles is shown. 

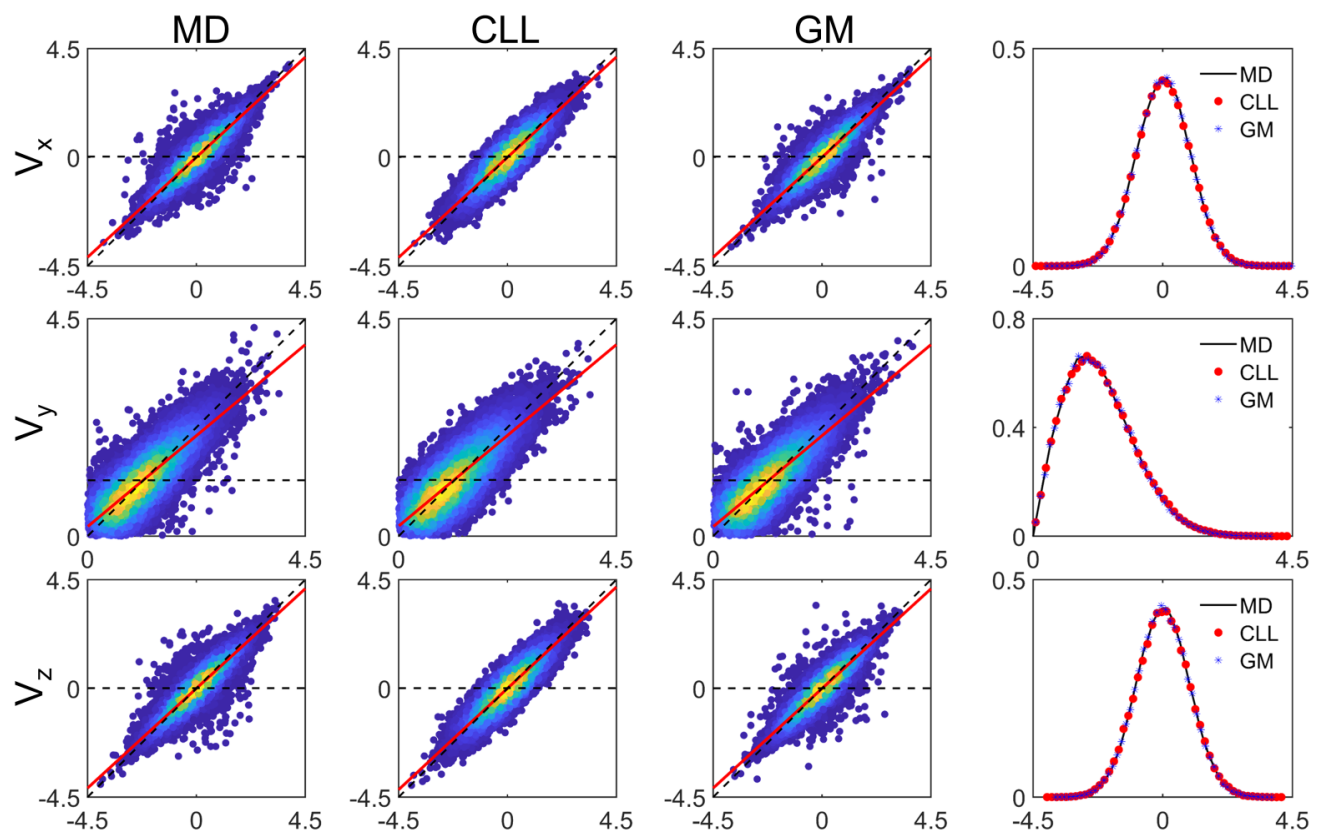

FIG. 22. Velocity correlations of impinging (x-axis) and reflected (y-axis) velocity components in $\mathrm{nm} / \mathrm{ps}$ for He-Au system at the top wall $\left(T_{b}=300 \mathrm{~K}, T_{t}=500 \mathrm{~K}, S_{w}=0\right)$. The dashed horizontal and diagonal lines indicate fully diffusive and specular conditions, respectively. Red lines indicate the least-square linear fit of the data. In the last column the corresponding probability density function for the reflecting particles has been shown.
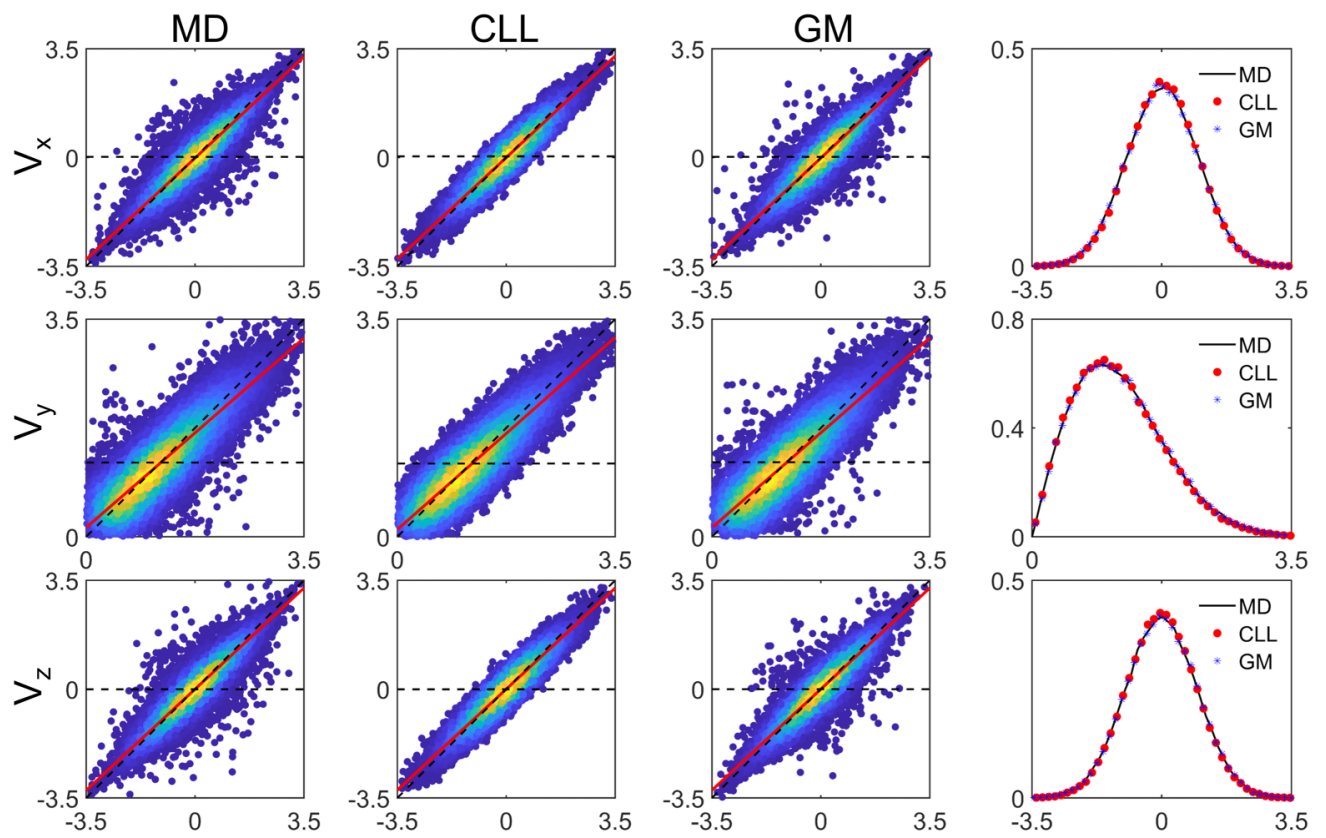

FIG. 23. Velocity correlations of impinging (x-axis) and reflected (y-axis) velocity components in $\mathrm{nm} / \mathrm{ps}$ for He-Au system at the bottom wall $\left(T_{b}=300 \mathrm{~K}, T_{t}=500 \mathrm{~K}, S_{w}=0.25\right)$. The dashed horizontal and diagonal lines indicate fully diffusive and specular conditions, respectively. Red lines indicate the least-square linear fit of the data. In the last column the corresponding probability density function for the reflecting particles has been shown. 

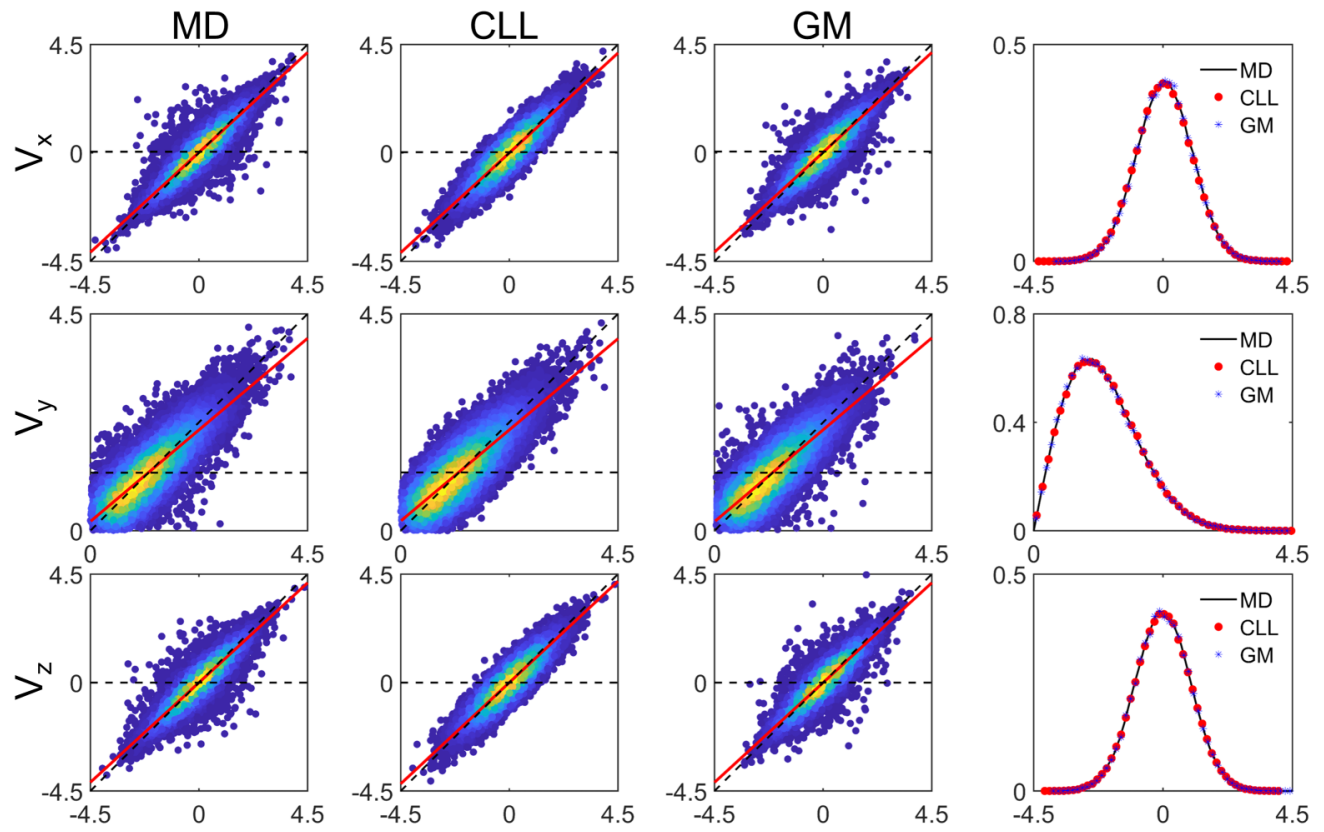

FIG. 24. Velocity correlations of impinging ( $x$ axis) and reflected ( $y$ axis) velocity components in $\mathrm{nm} / \mathrm{ps}$ for He-Au system at the top wall ( $T_{b}=300 \mathrm{~K}, T_{t}=500 \mathrm{~K}, S_{w}=0.25$ ). The dashed horizontal and diagonal lines indicate fully diffusive and specular conditions, respectively. Red lines indicate the least-square linear fit of the data. In the last column the corresponding probability density function for the reflecting particles has been shown.
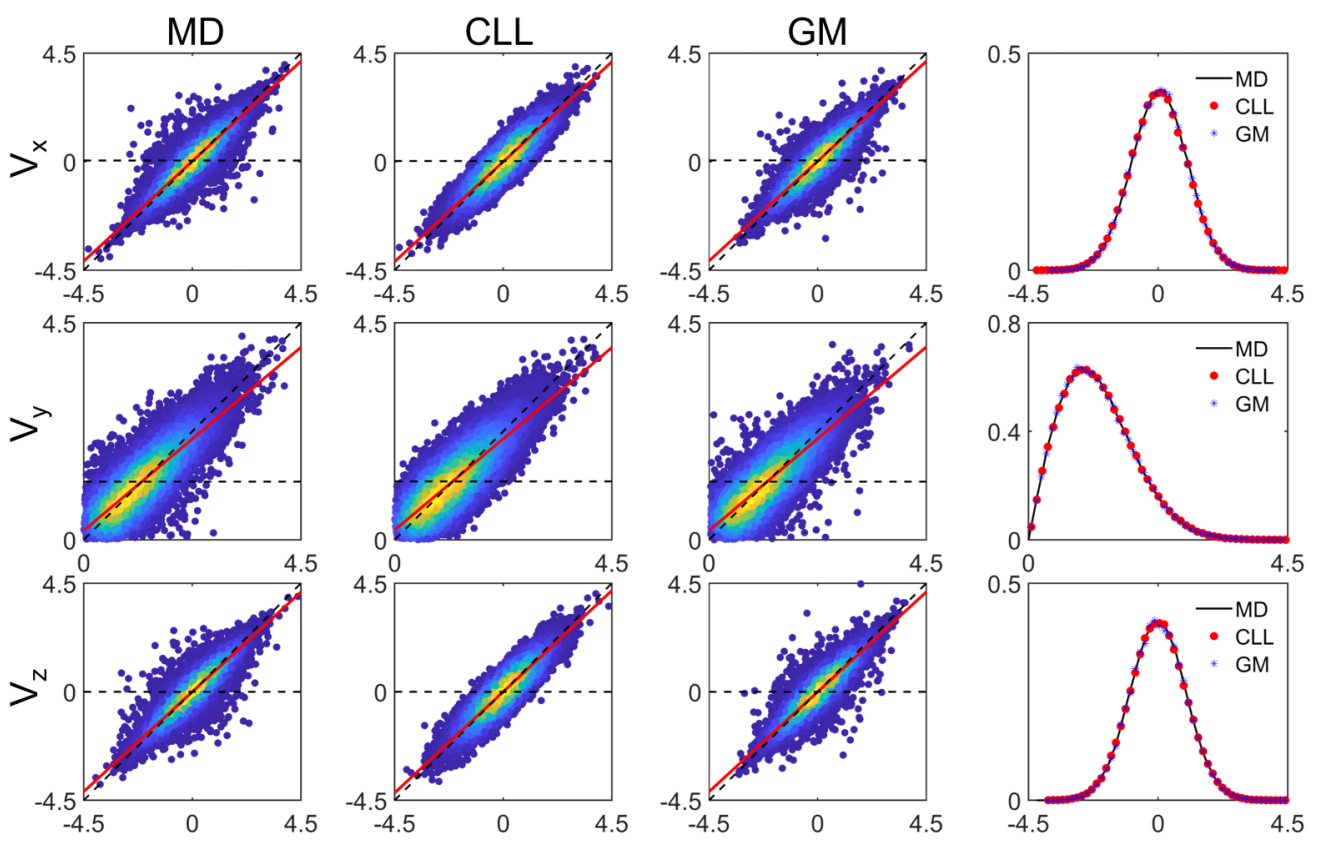

FIG. 25. Velocity correlations of impinging ( $x$ axis) and reflected ( $y$ axis) velocity components in $\mathrm{nm} / \mathrm{ps}$ for He-Au system at the bottom wall $\left(T_{b}=300 \mathrm{~K}, T_{t}=500 \mathrm{~K}, S_{w}=0.5\right)$. The dashed horizontal and diagonal lines indicate fully diffusive and specular conditions, respectively. Red lines indicate the least-square linear fit of the data. In the last column the corresponding probability density function for the reflecting particles has been shown.

[1] G. Karniadakis, A. Beskok, and N. Aluru, Microflows and Nanoflows: Fundamentals and Simulation (Springer Science \& Business Media, New York, 2006), Vol. 29.
[2] C. Shen, Rarefied Gas Dynamics: Fundamentals, Simulations and Micro Flows (Springer Science \& Business Media, New York, 2006). 
[3] S.-A. Kuhlmann, J. Reimann, and S. Will, On heat conduction between laser-heated nanoparticles and a surrounding gas, J. Aerosol Sci. 37, 1696 (2006).

[4] M. A. Gallis, D. J. Rader, J. N. Castaneda, J. R. Torczynski, T. W. Grasser, and W. M. Trott, Microscale rarefied gas dynamics and surface interactions for euvl and mems applications. Tech. Rep. No.: SAND2004-5329 (Sandia National Laboratories, 2004).

[5] G. A. Bird and J. Brady, Molecular Gas Dynamics and the Direct Simulation of Gas Flows (Clarendon, Oxford, 1994), Vol. 5.

[6] J. Zhang, Lattice boltzmann method for microfluidics: Models and applications, Microfluid. Nanofluid. 10, 1 (2011).

[7] H. Grad, On the kinetic theory of rarefied gases, Commun. Pure Appl. Math. 2, 331 (1949).

[8] J. C. Maxwell, Iii. on stresses in rarefied gases arising from inequalities of temperature, Proc. R. Soc. London 27, 304 (1878).

[9] C. Cercignani and M. Lampis, Kinetic models for gas-surface interactions, Transp. Theory Stat. Phys. 1, 101 (1971).

[10] R. Lord, Some extensions to the cercignani-lampis gas-surface scattering kernel, Phys. Fluids A 3, 706 (1991).

[11] W. W. Liou and Y. Fang, Microfluid Mechanics: Principles and Modeling (McGraw-Hill Education, New York, 2006).

[12] T. Liang, Q. Li, and W. Ye, Performance evaluation of maxwell and cercignani-lampis gas-wall interaction models in the modeling of thermally driven rarefied gas transport, Phys. Rev. E 88, 013009 (2013).

[13] S. C. Saxena and R. K. Joshi, Thermal Accommodation and Adsorption Coefficients of Gases (Hemisphere, New York, 1989).

[14] W. M. Trott, J. N. Castañeda, J. R. Torczynski, M. A. Gallis, and D. J. Rader, An experimental assembly for precise measurement of thermal accommodation coefficients, Rev. Sci. Instrum. 82, 035120 (2011).

[15] A. Agrawal and S. Prabhu, Survey on measurement of tangential momentum accommodation coefficient, J. Vac. Sci. Technol., A 26, 634 (2008).

[16] B.-Y. Cao, J. Sun, M. Chen, and Z.-Y. Guo, Molecular momentum transport at fluid-solid interfaces in mems/nems: A review, Int. J. Mol. Sci. 10, 4638 (2009).

[17] D. Bruno, M. Cacciatore, S. Longo, and M. Rutigliano, Gas-surface scattering models for particle fluid dynamics: A comparison between analytical approximate models and molecular dynamics calculations, Chem. Phys. Lett. 320, 245 (2000).

[18] A. Yakunchikov, V. Kovalev, and S. Utyuzhnikov, Analysis of gas-surface scattering models based on computational molecular dynamics, Chem. Phys. Lett. 554, 225 (2012).

[19] J. F. Padilla and I. D. Boyd, Assessment of gas-surface interaction models for computation of rarefied hypersonic flow, J. Thermophys. Heat Transfer 23, 96 (2009).

[20] K. Yamamoto, H. Takeuchi, and T. Hyakutake, Scattering properties and scattering kernel based on the molecular dynamics analysis of gas-wall interaction, Phys. Fluids 19, 087102 (2007).

[21] H. Struchtrup, Maxwell boundary condition and velocity dependent accommodation coefficient, Phys. Fluids 25, 112001 (2013).

[22] S. K. Dadzie and J. G. Méolans, Temperature jump and slip velocity calculations from an anisotropic scattering kernel, Physica A 358, 328 (2005).
[23] Q.-D. To, V.-H. Vu, G. Lauriat, and C. Léonard, Boundary conditions for gas flow problems from anisotropic scattering kernels, J. Math. Phys. 56, 103101 (2015).

[24] K. Yamamoto, H. Takeuchi, and T. Hyakutake, Characteristics of reflected gas molecules at a solid surface, Phys. Fluids 18, 046103 (2006).

[25] P. Spijker, A. J. Markvoort, S. V. Nedea, and P. A. J. Hilbers, Computation of accommodation coefficients and the use of velocity correlation profiles in molecular dynamics simulations, Phys. Rev. E 81, 011203 (2010).

[26] N. Yamanishi, Y. Matsumoto, and K. Shobatake, Multistage gas-surface interaction model for the direct simulation monte carlo method, Phys. Fluids 11, 3540 (1999).

[27] S. V. Nedea, A. J. H. Frijns, A. A. van Steenhoven, A. J. Markvoort, and P. A. J. Hilbers, Hybrid method coupling molecular dynamics and monte carlo simulations to study the properties of gases in microchannels and nanochannels, Phys. Rev. E 72, 016705 (2005).

[28] T. Liang and W. Ye, An efficient hybrid dsmc/md algorithm for accurate modeling of micro gas flows, Commun. Comput. Phys. 15, 246 (2014).

[29] N. A. Mehta and D. A. Levin, Molecular-dynamics-derived gas-surface models for use in direct-simulation monte carlo, J. Thermophys. Heat Transfer 31, 757 (2017).

[30] M. Liao, Q.-D. To, C. Léonard, and V. Monchiet, Nonparametric wall model and methods of identifying boundary conditions for moments in gas flow equations, Phys. Fluids 30, 032008 (2018).

[31] N. Andric, D. W. Meyer, and P. Jenny, Data-based modeling of gas-surface interaction in rarefied gas flow simulations, Phys. Fluids 31, 067109 (2019).

[32] M. Liao, Q.-D. To, C. Léonard, and W. Yang, Prediction of thermal conductance and friction coefficients at a solid-gas interface from statistical learning of collisions, Phys. Rev. E 98, 042104 (2018).

[33] G. W. Finger, J. S. Kapat, and A. Bhattacharya, Molecular dynamics simulation of adsorbent layer effect on tangential momentum accommodation coefficient, J. Fluids Eng. 129, 31 (2007).

[34] Y. A. Cengel and M. A. Boles, Thermodynamics: An Engineering Approach 6th Editon (SI Units) (McGraw-Hill, New York, 2007).

[35] H. W. Sheng, M. J. Kramer, A. Cadien, T. Fujita, and M. W. Chen, Highly optimized embedded-atom-method potentials for fourteen fcc metals, Phys. Rev. B 83, 134118 (2011)

[36] S. Mohammad Nejad, S. Nedea, A. Frijns, and D. Smeulders, The influence of gas-wall and gas-gas interactions on the accommodation coefficients for rarefied gases: A molecular dynamics study, Micromachines 11, 319 (2020).

[37] H. Sha, R. Faller, G. Tetiker, and P. Woytowitz, Molecular simulation study of aluminum-noble gas interfacial thermal accommodation coefficients, AIChE J. 64, 338 (2018).

[38] T. Mane, P. Bhat, V. Yang, and D. S. Sundaram, Energy accommodation under non-equilibrium conditions for aluminum-inert gas systems, Surf. Sci. 677, 135 (2018).

[39] K. Daun, T. Sipkens, J. Titantah, and M. Karttunen, Thermal accommodation coefficients for laser-induced incandescence sizing of metal nanoparticles in monatomic gases, Appl. Phys. B 112, 409 (2013). 
[40] M. Liao, R. Grenier, Q.-D. To, M. P. de Lara-Castells, and C. Léonard, Helium and argon interactions with gold surfaces: $\mathrm{Ab}$ initio-assisted determination of the he-au pairwise potential and its application to accommodation coefficient determination, J. Phys. Chem. C 122, 14606 (2018).

[41] S. Plimpton, Fast parallel algorithms for short-range molecular dynamics, J. Comput. Phys. 117, 1 (1995).

[42] S. A. Peddakotla, K. K. Kammara, and R. Kumar, Molecular dynamics simulation of particle trajectory for the evaluation of surface accommodation coefficients, Microfluid. Nanofluid. 23, 79 (2019).

[43] D. A. Reynolds and R. C. Rose, Robust text-independent speaker identification using gaussian mixture speaker models, IEEE Trans. Speech Audio Process. 3, 72 (1995).

[44] H. Permuter, J. Francos, and I. H. Jermyn, Gaussian mixture models of texture and colour for image database retrieval, in 2003 IEEE International Conference on Acoustics, Speech, and
Signal Processing, 2003, Proceedings (ICASSP'03) (IEEE, New York, 2003), Vol. 3, pp. III-569.

[45] H. Permuter, J. Francos, and I. Jermyn, A study of gaussian mixture models of color and texture features for image classification and segmentation, Pattern Recognition 39, 695 (2006).

[46] A. P. Dempster, N. M. Laird, and D. B. Rubin, Maximum likelihood from incomplete data via the em algorithm, J. R. Stat. Soc.: Ser. B (Methodol.) 39, 1 (1977).

[47] F. Pedregosa, G. Varoquaux, A. Gramfort, V. Michel, B. Thirion, O. Grisel, M. Blondel, P. Prettenhofer, R. Weiss, V. Dubourg et al., Scikit-learn: Machine learning in python, J. Mach. Learn. Res. 12, 2825 (2011).

[48] A. Frezzotti, S. Nedea, A. Markvoort, P. Spijker, and L. Gibelli, Comparison of molecular dynamics and kinetic modeling of gas-surface interaction, in AIP Conference Proceedings (American Institute of Physics, Melville, NY, 2008), Vol. 1084, pp. 635-640. 